\title{
An fMRI-Constrained MEG Source Analysis with Procedures for Dividing and Grouping Activation
}

\author{
Norio Fujimaki,* Tomoe Hayakawa,* Matthew Nielsen,*,1 Thomas R. Knösche,† and Satoru Miyauchi* \\ *Brain Function Laboratory, Communications Research Laboratory, 588-2 I waoka, I waoka-cho, Nishi-ku, Kobe 651-2492, J apan; and \\ †Max Planck Institute of Cognitive Neuroscience, Stephanstrasse 1a, P.O. Box 500355, D-04303 Leipzig, Germany
}

Received November 12, 2001

To analyze neural activity using magnetoencephalography (MEG) and functional magnetic resonance imaging ( $f M R I$ ), we developed a method for fixing equivalent current dipoles of MEG in activation areas of fMRI. It includes a procedure for dividing large fMRI activation volumes into subvolumes in each of which a dipole is placed and another procedure for grouping neighboring dipoles whose temporal changes are inseparable based on MEG data. To optimize the procedures' parameters, we carried out simulations and found that (1) any single dipole within $10 \mathrm{~mm}$ from a true source can explain ME G data with a correlation of $94 \%$ on average for the low signal-to-noise ratio of 3 and (2) a neighboring dipole within a few tens of millimeters from the dipole nearest to the true source tends to be highly incorporated in explaining MEG data. We applied the method to data measured in a language experiment and detected 13 significant sources. The results show that the present method is promising for detecting neural activity originating from a number of separate neural sources. 2002 Elsevier Science (USA)

Key Words: ME G; inverse problem; fMRI; constraint; neural source; dipole; cross talk; criteria; grouping; language.

\section{INTRODUCTION}

Magnetoencephalography (MEG) has high temporal resolution and is a strong tool for noninvasive measurements of human brain functions. Since solutions cannot be uniquely determined, various constraints have been imposed on the inverse problems of MEG (Hämäläinen et al., 1993). In many applications, mathematical constraints have been used: the number of equivalent current dipoles, which we simply call "dipoles" in this report, was assumed to be one to several,

\footnotetext{
${ }^{1}$ Current address: MR Group, Siemens-Asahi Medical Technologies Ltd., 3-20-14, Higashi-gotanda, Shinagawa-ku, Tokyo 141-8644, J apan.
}

and iterative calculations were used to fit locations and moments of the dipoles to MEG data (Sarvas, 1987; Scherg and Berg, 1991). Especially the single-dipole approximation has been empirically recognized to be sufficient for early components of neural activity related to presented stimuli, but not for many of the late components (Fujimaki et al., 1998). Although use of plural dipoles improves the latter fittings, it enhances a tendency for iterative calculations with different initial conditions to converge to different solutions, i.e., we are troubled by nonuniqueness of the solution. In an opposite extreme within possible mathematical constraints, many dipoles were placed so that single dipoles were fixed at every mesh point inside a brain and their moments were fitted to MEG data. Since the number of unknown dipole parameters usually exceeded that of independent components in MEG data, additional constraints such as a minimum norm condition have been imposed (Wang et al., 1992). This method has an advantage that a solution can be obtained by a pseudo-inverse matrix without iterative calculations, but has a drawback of showing source distributions shallower and wider than the actual source when a focal source is assumed (Sekihara and Scholtz, 1996). Although it can be improved by using artificial weights for source depths (Grave de Peralta Menendez et al., 1997; I waki and Ueno, 1998) or by using another norm definition and limitation on the number of dipoles (Matsuura and Okabe, 1995), we are not sure how the mathematical assumptions match with actual neural activation. Therefore, mathematical constraints alone are insufficient to detect distributed neural sources.

In the other approaches, anatomical and physiological constraints have been used; possible dipole locations were restricted or weighted in gray matter and in activation areas detected by functional magnetic resonance imaging (fMRI) (Dale et al., 1993, 2000; George et al., 1995; Ahlfors et al., 1999; Korvenoja et al., 1999; Wagner and Fuchs, 1999) or positron emission tomography (Heinze et al., 1994). F urthermore, an additional constraint was given to orient dipole moments in a 
direction perpendicular to cortical surfaces (Liu et al., 1998).

The methods for applying fMRI constraints proposed previously are classified into two categories: one method is to place dipoles at all mesh points in a brain or on cortical surfaces with a priori probabilities depending on fMRI activation (Wagner and Fuchs, 1999; Dale et al., 2000), and the other is to place dipoles in fMRI activation foci (Ahlfors et al., 1999; Korvenoja et al., 1999). For either case, dipole locations are fixed and their moments are fitted to MEG data. The methods in the first category have the advantage that $\mathrm{FMRI}$ invisible dipoles, i.e., dipoles located in areas where fMRI did not show activation, can be obtained on the condition that nonzero probabilities are given (Liu et al., 1998). However, if we permit such a possibility of having dipoles at every mesh point, the number of unknown parameters exceeds that of independent components in MEG data, because the former is typically more than a few thousand, while the latter is less than a few hundred, the number of sensors implemented in modern MEG systems. Since other mathematical constraints such as the minimum norm condition are required again, there remains an ambiguity of whether the obtained solutions are the ones that we look for or not.

Although the methods in the second category cannot show $\mathrm{fMRI}$-invisible dipoles, they have the advantage that dipoles can be uniquely determined by minimizing the squares of differences between measured and calculated magnetic fields, assuming the number of unknown dipole parameters is smaller than that of independent components in MEG data. In two reports (Ahlfors et al., 1999; Korvenoja et al., 1999), single dipoles were placed at each focus of fMRI activation; however, this is not adequate when the activation has large spatial extent. Besides, from fMRI activation we know only the possible spatial extent, not the current distribution, because fMRI signals reflecting hemodynamic changes are not necessarily proportional to electrical currents of neural sources. Thus, it is necessary to consider how many and where dipoles should be placed in a volume of $\mathrm{FMRI}$ activation.

Furthermore, we need to take into account a phenomenon called "cross talk": when we fix plural dipoles and fit their moments to magnetic fields produced by a true source, the magnetic fields can be mainly explained by the dipole nearest to the source, but a part of them can also be explained by the other dipoles. Their average contributions were evaluated using a Monte Carlo simulation method (Liu et al., 1998); however, the characteristics strongly depend on spatial direction so that they need to be evaluated explicitly for detailed consideration.

In the present report, we carried out simulations to find a method of placing dipoles only in fMRI activation volumes. The purposes of the simulations were to learn
(1) how to place dipoles from knowledge of spatially distributed fMRI activation and (2) how cross talk depends on dipoles' locations relative to each other and to the true source. Direction-dependent characteristics of fittings were also clarified from these simulations. Further, we obtained criteria from the simulation results and proposed an fMRI-constrained MEG source analysis method incorporating procedures for dividing fMRI active regions and grouping inseparable MEG dipoles. This was applied to a set of $\mathrm{FMRI}$ and MEG data measured from one subject using a language task.

\section{SIMULATIONS}

In the following simulations, we used source analysis software (ASA Version 2.1; ANT Software B.V.) and assumed a head shape approximated by a sphere with a radius of $75 \mathrm{~mm}$ and uniform conductivity and the sensor layout of a 148-channel whole-head MEG system (Magnes $2500 \mathrm{WH}$; Biomagnetic Technologies, Inc.) (Fig. 1a).

\section{Neural Source Models}

Real neural sources are either focal or distributed on cortical surfaces. We modeled them by using three types of representative sources: a point source (meaning a single dipole), a linear array of dipoles, and a square array of dipoles (Fig. 1a). We call them "source" dipoles or true "sources" in this report in contrast to the constrained dipoles to be used in the reconstruction method. An actual biological source could be described as a mixture of these model sources. We varied the depth of the source centers to be 10,30 , and $50 \mathrm{~mm}$, because most areas of neural activation that contribute to MEG are closer to the brain surface than $50 \mathrm{~mm}$. Since MEG data are insensitive to radial currents, moments of source dipoles were oriented in a tangential plane. Furthermore, from the physiological knowledge that neural currents flow in the direction perpendicular to cortical surfaces, MEG sources mainly exist in cerebral sulci. Thus, the dipole arrays in our model were arranged on a plane perpendicular to the tangential plane; a linear array was arranged on a line along the tangential and radial directions. The line lengths of the linear arrays and side lengths of the square arrays were varied to be 20,40 , and $60 \mathrm{~mm}$, as long as they remained inside the brain compartment of the spherical head model. The arrays consisted of dipoles that had the same moment and were separated from each other by $5 \mathrm{~mm}$. The $5-\mathrm{mm}$ spacing was chosen as the result of comparing it with the sufficiently dense spacing of $1 \mathrm{~mm}$ in typical simulations of fitting a constrained dipole; it showed differences less than 0.0005 $(0.05 \%)$ for $\mathrm{CC}_{m}, 0.002$ for normalized moment magnitude, and $0.02^{\circ}$ for projected angular error. The meanings of the above quantities are described under Loca- 


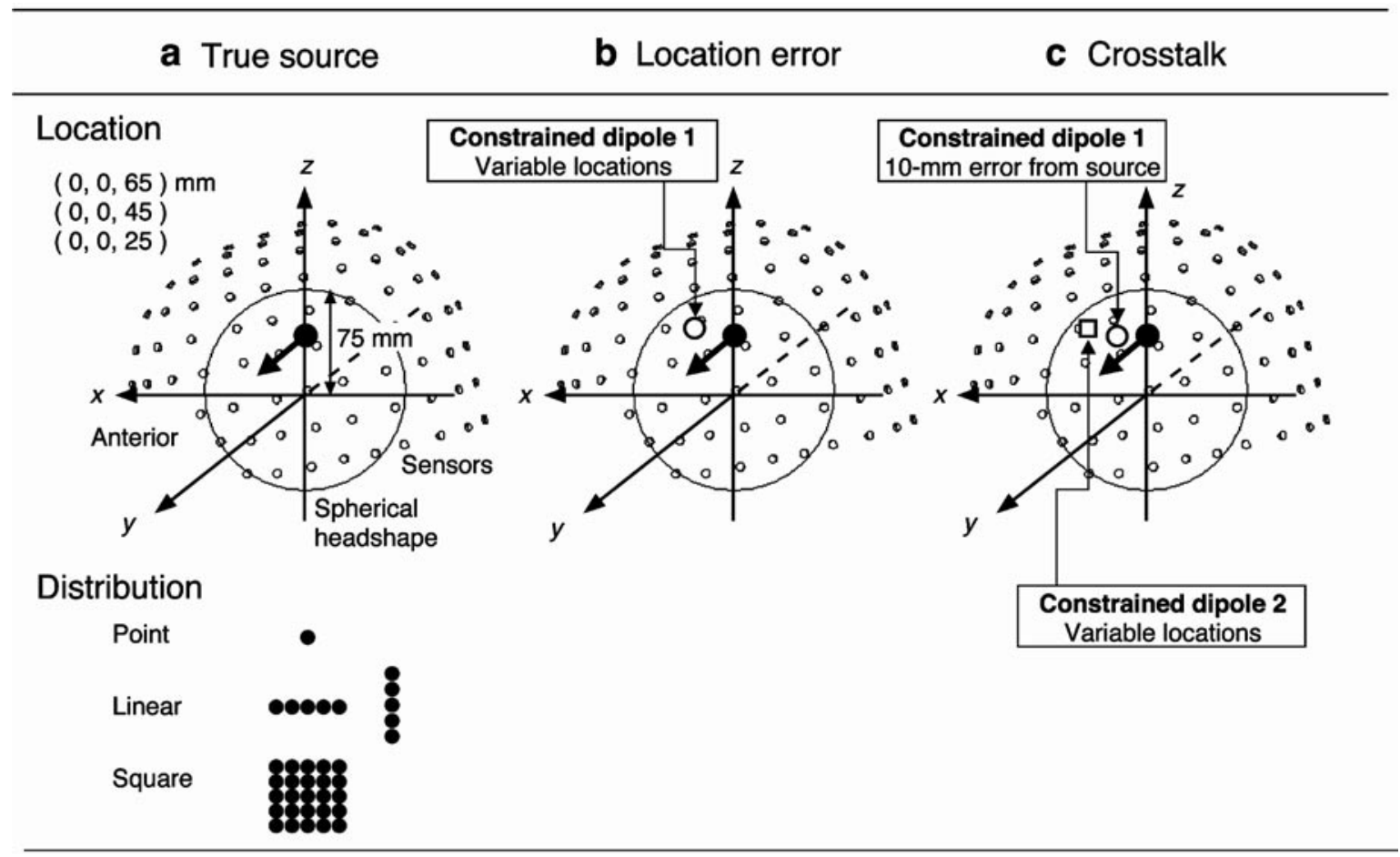

FIG. 1. (a) Source location in the medial central area of the brain, a head shape approximated by a sphere with a radius of $75 \mathrm{~mm}$, the 148-channel sensor layout (top), and three source types (bottom) used in simulations. (b) A scheme of simulations for location error in which a single location-constrained di pole 1 was fitted to magnetic fields produced by the sources. (c) A scheme of simulations for cross talk in which constrained dipoles 1 and 2 were fitted to the magnetic fields.

tion Error and Effects of Asymmetrical Sensor Layout in the Fittings, respectively.

The simulations were carried out in detail for sources that had a leftward moment and were located in the medial central area (Fig. 1a). In order to examine differences due to asymmetry in sensor layout near frontal and lower areas, we also carried out simulations for limited cases using four additional source areas (Table 1). The moment orientations were chosen to be two perpendicular directions on tangential planes in these areas.

\section{Location Error}

In this section, we estimated how large an error in location of a constrained dipole is permitted for good fittings. First, we calculated the magnetic field produced by a particular modeled source and added

TABLE 1

Location, Depth, and Moment Orientation of the Sources U sed in the Simulations

\section{Area}

Medial central area

Medial prefrontal area

Left inferior frontal area

Left superior temporal area

Medial occipital area
Location for three depths 10,30 , and $50 \mathrm{~mm}$

$(0,0,65 / 45 / 25)$

$(65 / 45 / 25,0,0)$

$(46 / 32 / 18,46 / 32 / 18,0)$

$(0,65 / 45 / 25,0)$

$(-65 /-45 /-25,0,0)$

Orientation of moment

Left: $(0,1,0)$

Upper: $(0,0,1)$

Left: $(0,1,0)$

Upper: $(0,0,1)$

Anterior-right: $(0.71,-0.71,0)$

Upper: $(0,0,1)$

Anterior: $(1,0,0)$

Upper: $(0,0,1)$

Left: $(0,1,0)$

Note The coordinate system is described in Fig. 1a. Detailed locations are given for five areas: the medial central area near the frontoparietal lobe, the supplementary motor area, and the cingulate gyrus; the medial prefrontal area near Brodmann's areas 9, 10, 32, and 33; the left inferior frontal area near Broca's area; the left superior temporal area near the auditory area and Wernicke's area; and the medial occipital area near the calcarine fissure. 

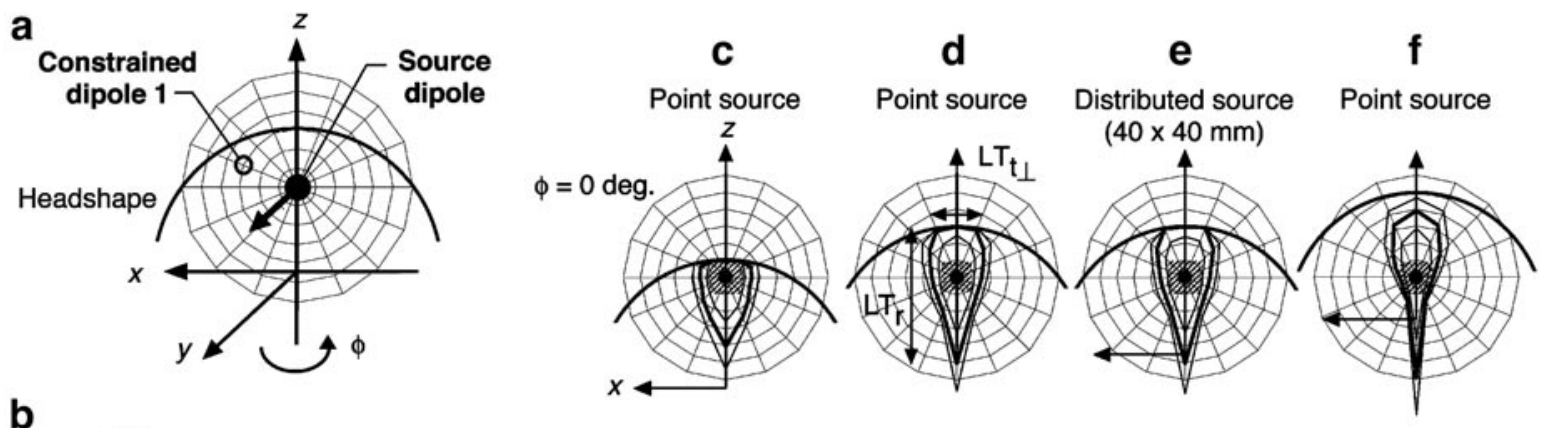

b
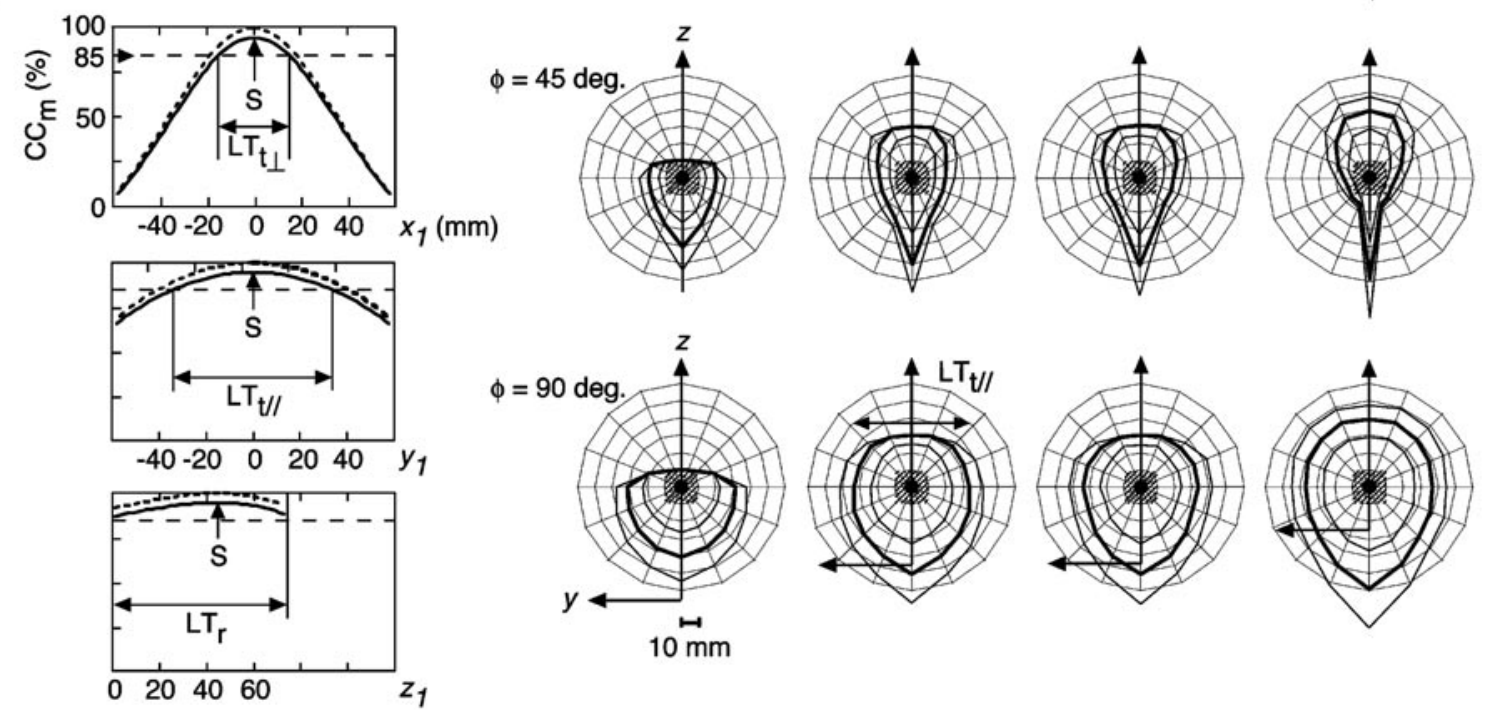

FIG. 2. (a) Directions in which location of constrained dipole 1 was varied. (b) An example of simulated correlation coefficient (CC $\left._{m}\right)$ dependence on location for constrained dipole $1,\left(x_{1}, y_{1}, z_{1}\right)$, in which a y-oriented point source with a 30-mm depth was assumed. Solid curves show $\mathrm{CC}_{\mathrm{m}}$ for the signal-to-noise ratio of 3 , while dashed curves show $\mathrm{CC}_{\mathrm{m}}$ without noise. The symbol $\mathrm{S}$ denotes location of the source, and LT denotes location tolerance region in which the $\mathrm{CC}_{m}$ value was higher than $85 \%$. The suffixes mean the directions al ong which the location tolerance region was evaluated (Table 2). (c-f) Simulated boundaries of the location tolerance region in planes with angle $\phi$ of 0,45 , and $90^{\circ}$ (thick curves). Outer and inner thin curves show the boundaries at which the $\mathrm{CC}_{\mathrm{m}}$ threshold was 80 and $90 \%$. The true sources were located on the $z$ axis and oriented in the y direction, and their depths were $10 \mathrm{~mm}$ for (c), $30 \mathrm{~mm}$ for (d) and (e), and $50 \mathrm{~mm}$ for (f) in the medial central area. They were a point source for (c), (d), and (f) and a source distributed uniformly on a $40 \times 40$-mm square for (e).

Gaussian random noise to make a signal-to-noise ratio of 3 , when the signal-to-noise ratio was defined to be the square root of the ratio of the power (i.e., the square root of the ratio of the sum of the squares for a signal at all channels to that for noise). The value of 3 is the minimum signal-to-noise ratio for meaningful source localization in our experience. Then, we placed a single location-constrained dipole 1 (Fig. 1b) and fitted its moment to the magnetic field to evaluate the correlation coefficient between the magnetic fields produced by the true source and those produced by the constrained dipole $1\left(C_{m}\right.$, see Appendix $\left.A\right)$. Fitting and evaluation of $\mathrm{CC}_{\mathrm{m}}$ were carried out for all possible combinations of source depth and distribution type for each location of dipole 1 in 2-mm steps along each of 114 directions within the head model. The directions were determined in the following way (F ig. 2a): first, 16 directions were determined so that they divided the $x-z$ plane around a true source into $22.5^{\circ}$ steps; then the other directions were obtained by rotating the 16 directions around the $z$ axis in $22.5^{\circ}$ steps. Simulations were repeated 10 times for each condition to average over noise. The 10-time averaging was sufficient because standard errors were less than $0.007(0.7 \%)$ for $\mathrm{CC}_{\mathrm{m}}, 0.02$ for normalized moment magnitude, and $1.0^{\circ}$ for the projected angular error among the data for all sources (Table 1) on the condition that $\mathrm{CC}_{\mathrm{m}}$ was more than $85 \%$ and the constrained dipole 1 satisfied the criterion (LC). The meanings of the latter two quantities are described under Effects of Asymmetrical Sensor Layout in the Fittings and that of the criterion (LC) under Location Criterion.

Simulation results showed that $\mathrm{CC}_{\mathrm{m}}$ values became smaller as the location of dipole 1 was moved farther from the true source location (Fig. 2b). The ratio of $\mathrm{CC}_{m}$ values with a signal-to-noise ratio of 3 (solid curves) to those without noise (dashed curves) was about 95\%, which agreed well with a theory assuming a random 


\section{TABLE 2}

Symbols That Show Directions for Location Error of Constrained Dipoles

\begin{tabular}{ll}
$\mathrm{t} \perp$ & $\begin{array}{c}\text { Direction perpendicular to source moment in the } \\
\text { tangential plane at the true source location } \\
\text { Direction parallel to source moment in the } \\
\text { tangential plane at the true source location } \\
\text { (Positive or negative) radial direction at the true } \\
\text { source location }\end{array}$ \\
\hline
\end{tabular}

noise distribution (Appendix A). We determined a "location tolerance region" in which $\mathrm{CC}_{m}$ was higher than $85 \%$. The thick curves in Figs. 2c-2f show boundaries of the location tolerance region where a y-oriented source dipole was assumed in the medial central area, while the thin curves show the boundaries at which $\mathrm{CC}_{\mathrm{m}}$ was 80 and $90 \%$. The extent of the location tolerance region in the case of a point source ( $\mathrm{Fig} .2 \mathrm{~d}$ ) resembled that in the case of a source distributed on a $40 \times 40-\mathrm{mm}$ square (Fig. 2e) and was smallest in the $x$ direction and largest in the $z$ direction for all source depths of 10, 30, and $50 \mathrm{~mm}$ (Figs. 2c, 2d, and 2f). In other words,

$$
\mathrm{LT}_{\mathrm{t} \perp}<\mathrm{LT}_{\mathrm{t} \|}<\mathrm{LT} \mathrm{T}_{\mathrm{r}}
$$

where LT denotes the extent along the directions of location errors shown by the suffixes that are defined in Table 2. Relationship (1) is valid not only for the sources in the medial central area, but also for those at the other four source areas with either of two orthogonal moments (Table 1). It shows how well constrained dipoles can be fitted even when a location error exists; the fitting quality, $C_{m}$, is most sensitive to location errors in the $(t \perp)$ direction and is least sensitive to location errors in the radial direction.

A previous simulation showed that direction-dependent confidence limits of locations for fitted single dipoles had the same direction dependence as Eq. (1) (Hari et al., 1988). This agreement is reasonable because the meaning of LT is similar to that of the confidence limit, which denotes how large a location error is permitted to be to keep the difference in magnetic fields less than the noise level. Furthermore, the present simulation results (Figs. 2c-2f) are consistent with the results of another study (Uusitalo and IImoniemi, 1997) in which the authors simulated an angle between the two dipoles in measurement space and estimated that the minimum detectable separation length between them was $2 \mathrm{~cm}$ in the $(\mathrm{t} \perp)$ direction and $4 \mathrm{~cm}$ in the $(\mathrm{t} \|)$ and $(\mathrm{r})$ directions. This means that magnetic fields changed most rapidly in the $(t \perp)$ direction.

\section{Location Criterion}

Hatched areas in Figs. 2c-2f show a spatial extent within $10 \mathrm{~mm}$ from the true source along the tangen- tial and radial directions, in other words, a cylinder with a circular side having a $20-\mathrm{mm}$ diameter on a tangential plane and a $20-\mathrm{mm}$ side length along a radial direction. It is included inside the boundaries of the location tolerance region (thick curves) in the depth range of 10 to $50 \mathrm{~mm}$ for all cases in the simulation. Thus, we obtained the following location criterion:

(LC) any single dipole within $10 \mathrm{~mm}$ from the true source along the tangential and radial directions can explain the magnetic fields produced by the source with a correlation $\left(\mathrm{CC}_{\mathrm{m}}\right)$ of $85 \%$ or better.

This suggests that if an $\mathrm{FMRI}$ activation volume has a spatial extent of less than $20 \mathrm{~mm}$, a $\mathrm{CC}_{\mathrm{m}}$ of higher than $85 \%$ will be obtained by placing a single dipole at its center. If the volume is larger, we can divide it so that each subvolume has an extent smaller than $20 \mathrm{~mm}$, and thus the neural activation in the subvolume can be represented by a single dipole placed at its center. It should be remarked that $85 \%$ was the minimum value and the mean $\mathrm{CC}_{\mathrm{m}}$ was much higher, as described in the next section. In this respect, if we vary the location criterion length along the tangential and radial directions like the shape of the boundaries (Figs. 2c-2f) instead of the constant value $(10 \mathrm{~mm})$, the subvolume is considered to be made larger than the cylinder described above. However, it is not easy for the following three reasons: (1) for the tangential plane, it would be necessary to determine the orientation of the unknown source moment, e.g., from anatomical information; (2) for the radial direction, we may use the longer criterion length for the sources located deeper; however, the definition of the radial direction is unclear for the deep sources in a real headshape; (3) in either direction, it would be difficult to divide the activation volumes into nonoverlapped subvolumes if the subvolume has a complex shape.

\section{Effects of Asymmetrical Sensor Layout in the Fittings}

To examine the precision of fitted dipole parameters in comparison with source parameters and their variations due to asymmetry in sensor layout near frontal and lower areas, we carried out simulations using nine conditions of source area/orientation (Table 1) in the same way as those in the medial central area described under Location Error. We focused on the three quantities $\mathrm{CC}_{m}$, normalized moment magnitude, and projected angular error. The normalized moment magnitude means a moment magnitude of constrained dipole 1 divided by that of the true source moment, and the projected angular error means an angle between the moment of the true source and that of dipole 1 projected on the source's tangential plane. This definition of the projected angular error was intended to ignore the change of angle due to the change of tangential plane from the source to dipole 1. For the purpose of representing the true source by the constrained dipole, 
it is better that the latter moment is nearer to the former one, i.e., $\mathrm{CC}_{\mathrm{m}}$ and the normalized moment magnitude are nearer to 1 , and the projected angular error is nearer to 0 .

The simulations described in this section were carried out with two restrictions: (1) that only point sources were examined and (2) that the locations of constrained dipoles were varied along each of 26 directions, i.e., twice as coarse in the angle step as under "Location Error" to reduce computation time. The first restriction is justified based on a finding that indicated there was a close resemblance between the three quantities for point sources and those for all sources; mean (standard deviation (SD)) of $\mathrm{CC}_{\mathrm{m}}$, normalized moment magnitude, and projected angular error were 94(2)\%, $1.00(0.17)$, and $1.29(0.36)^{\circ}$, respectively, for point sources, while they were $94(2) \%, 0.98(0.16)$, and $1.28(0.34)^{\circ}$ for all source types (i.e., the mean of these quantities over all source types, including point sources). The second restriction is justified based on a finding that indicated that the difference in the means of $\mathrm{CC}_{\mathrm{m}}$, normalized moment magnitude, and projected angular error was less than $0.004(0.4 \%), 0.01$, and $0.1^{\circ}$ between angle steps of 45 and $22.5^{\circ}$. The three quantities in the above two findings were obtained by averaging across the three source depths in the medial central area and constrained dipole 1 that satisfied the LC.

We carried out a nonparametric Kruskal-Wallis test implemented in statistical software (StatView V5.0, SAS Institute, Inc.) for the three quantities at 4461 locations of dipole 1 that satisfied the LC. They were tested for two factors: nine combinations of area/orientation for sources and three source depths (Table 1 ). The $\mathrm{CC}_{\mathrm{m}}(\mathrm{P}<0.0001)$ and projected angular error $(\mathrm{P}<$ $0.0001)$ differed significantly among the source area/ orientation conditions, but the normalized moment magnitude did not. Among the three source depths, $\mathrm{CC}_{\mathrm{m}}(\mathrm{P}<0.0001)$, normalized moment magnitude $(\mathrm{P}<$ $0.0001)$, and projected angular error $(P<0.01)$ differed significantly.

Although the statistical test showed significant differences for almost all contrasts, the differences in the means of the three quantities were generally small (Table 3). Thus, we need not change the location criterion nor pay attention to the differences in the fitted dipole parameters among the brain areas. Among the small differences, the projected angular error showed a comparatively large difference in the medial prefrontal and left inferior frontal areas. A possible reason for the difference was a lack of sensors in the anterior areas, which was necessary to make an opening for the subject's face. Given this, the results imply that the projected angular error is more sensitive to the lack of magnetic-field data than $\mathrm{CC}_{m}$ and the normalized moment magnitude. Since the location tolerance region strongly depends on directions (Figs. 2c-2f), the grand average of $\mathrm{CC}_{\mathrm{m}}$, which was obtained by an averaging
TABLE 3

Grand Average (SD) and Maximum Difference in the Means of the Three Quantities among the Source Conditions

\begin{tabular}{lccc}
\hline & & \multicolumn{2}{c}{$\begin{array}{c}\text { Maximum difference } \\
\text { in means among } \\
\text { source conditions }\end{array}$} \\
\hline & $\begin{array}{c}\text { Grand average } \\
\text { (SD) }\end{array}$ & Area/orientation & Depth \\
\hline $\begin{array}{l}\mathrm{CC}_{\mathrm{m}}(\%) \\
\text { Normalized moment } \\
\text { magnitude }\end{array}$ & $94(2)$ & $1.0^{* *}$ & $0.8^{* *}$ \\
$\begin{array}{c}\text { Projected angular } \\
\text { error (degrees) }\end{array}$ & $1.73(0.92)$ & 0.016 & $0.017^{* *}$ \\
\hline
\end{tabular}

Note Data were averaged across locations of dipole 1 that satisfied the location criterion. Single and double asterisks denote that statistical tests showed significant differences among the conditions ( $P<0.01$ and $\mathrm{P}<0.0001$, respectively).

across dipoles that satisfied the LC and all source conditions, was 94\% (Table 3), far beyond the minimum value of $85 \%$ that the criterion ensures.

The other quantity, goodness of fit, has also been widely used to express how well the fields produced by the fitted dipoles match to the measured fields. Since the goodness of fit is approximately the squared $\mathrm{CC}_{\mathrm{m}}$ in many cases, a $\mathrm{CC}_{\mathrm{m}}$ of $94 \%$ corresponds to a goodness of fit of $88 \%$. The fitting quality shown by these figures was considered sufficient from our experience in analyzing MEG data related to higher cognitive brain functions. However, one might prefer the other threshold value. Figure 3 shows the dependence of the grand average $\mathrm{CC}_{\mathrm{m}}$ on the location criterion length (circles). If we increase the length, the expected $C_{m}$ becomes lower, resulting in worse fittings. On the other hand, if we decrease the length, $\mathrm{CC}_{\mathrm{m}}$ is not drastically improved, while the number of dipoles increases. To estimate the number of dipoles as a function of the spatial distribution of the fMRI activation, we assumed three ideal cases with one (linear), two- (square), and three(cubic) dimensional distributions of $328 \mathrm{fMRI}$ activation voxels $(4 \times 4 \times 4 \mathrm{~mm})$ and calculated the theoretical number (triangular marks in Fig. 3) as 328 (4/ $L C L)^{D}$, where $L C L$ denotes location criterion length and $D$ dimension. The number of voxels was taken from the typical fMRI activation data used under Application. The curve with square marks shows the number of dipoles extracted from the actual distribution of the data. It resembled the two-dimensional theoretical curve. Since the maximum number of dipoles whose moments can be uniquely determined from 148 MEG sensor data is less than 74 (broken line in Fig. 3) as described later (Results and Discussion under Application), a location criterion length of about $10 \mathrm{~mm}$, which gives a number slightly less than the limit, is the best choice. 


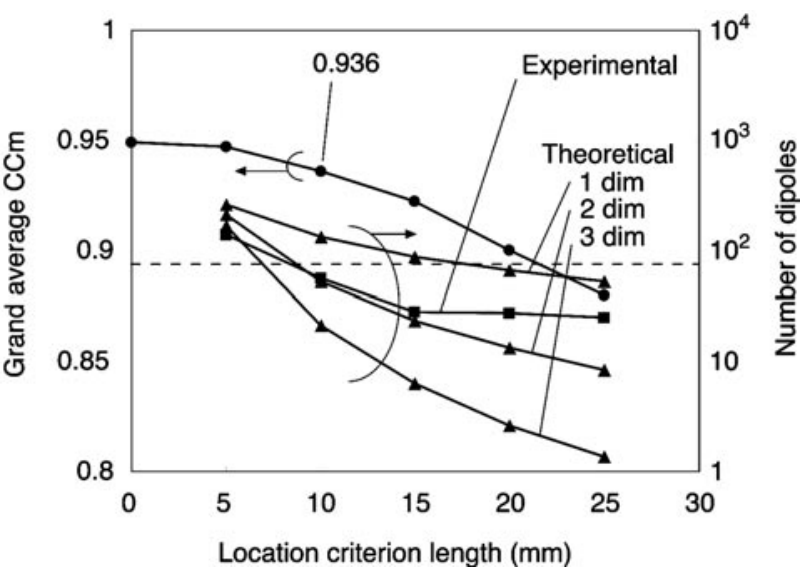

FIG. 3. Dependence of the grand average $\mathrm{CC}_{\mathrm{m}}$ on location criterion length in the tangential and radial directions (circles). The $\mathrm{CC}_{\mathrm{m}}$ values simulated under Effects of Asymmetric Sensor Layout in the Fittings were averaged across the cases in which dipole 1 was located inside the criterion length in either the tangential or the radial direction for nine source area/orientation conditions and three source depth conditions. The 10-mm length is chosen in the LC. The other curves denote the experimental (squares) and theoretical (triangles) numbers of dipoles obtained from the data used under Application. The "1-3 dim" denotes the curves calculated assuming 328 voxels distributed in one to three-dimensional distributions. The broken line denotes the maximum number of dipoles (74) whose moments can be uniquely determined from 148 MEG sensor data.

\section{Cross Talk}

Based on the findings described under Location Criterion, we divide large $\mathrm{fMRI}$ activation volumes to match the LC and place a constrained dipole in each subvolume to represent any source current distribution inside the subvolume in our method. The dipoles are then fitted to the magnetic fields produced by the sources. This process is carried out under the overdetermined condition if the number of dipole parameters is less than that of measured data (Wang et al., 1992). Therefore, if the true source is focal like a point source and happens to be at the same location as the constrained dipole, the magnetic field produced by the source is completely explained by the dipole. Otherwise, it cannot be fully explained by the dipole; neighboring dipoles must be incorporated to explain a part of the magnetic fields, i.e., cross talk occurs. In this section, we evaluate how far dipoles need to be placed apart from each other to keep cross talk low.

The simulation in this section was similar to that for location error, except that we placed two location-constrained dipoles, dipole 1 and dipole 2 (F ig. 1C), and fitted their moments to magnetic fields produced by the true sources. Then, we evaluated a cross talk value defined by

$$
\text { cross talk }=\frac{\left|\mathbf{q}_{2}\right|}{\left|\mathbf{q}_{1}\right|},
$$

where $\left|\mathbf{q}_{1}\right|$ and $\left|\mathbf{q}_{2}\right|$ denote the moment magnitudes of dipoles 1 and 2 . It should be remarked that this defi- nition of cross talk differs from that of the reference (Liu et al., 1998) in which cross talk was defined as the ratio of the squared moment magnitudes of dipoles located at mesh points having no source to those of dipoles located at mesh points having sources. The other difference is that 5 to 20 randomly located sources were used to eval uate average cross talk in the reference while only one source was used in the present simulations.

In simulating cross talk, dipole 1 was intentionally shifted by $10 \mathrm{~mm}$, the distance allowed by the LC, along the tangential or radial direction from true sources. The location of dipole 2 was varied by $2-\mathrm{mm}$ steps in each of 114 directions around dipole 1 (Fig. 4a) in the same way as constrained dipole 1 under Location Error. Simulation results showed that cross-talk values gradually decreased as dipoles 1 and 2 were further separated, except that sharp jumps appeared in the cross-talk curves (Fig. 4b). The fitted dipoles are illustrated in Fig. 5 for the case shown in the top of Fig. $4 b$. The jumps were due to a truncating process for singular value decomposition in solving the inverse solutions, which were intended to inhibit divergence in calculating the inverse matrix when plural dipoles were placed in the neighborhood. They occurred when dipole 2 was shifted from dipole 1 by several to $20 \mathrm{~mm}$. Although the major results cited in this report do not seriously change, the tolerance values described below may be affected by changing the threshold value of the truncation. The cross-talk values diverged at the point indicated by the symbol $\mathrm{S}$ in the top of Fig. 4b. This is because the moment magnitude of dipole $1,\left|\mathbf{q}_{1}\right|$, became 0 at that point (Fig. 5). The difference between the cross-talk curves with (solid curves) and without (dashed curves) noise was so small that we did not include noise in the simulation of cross talk thereafter.

We defined high (low) cross talk as a cross-talk value higher (lower) than 50\%. We focused on the spatial extent of low cross talk within a spherical headshape and named it the "separation tolerance region." If there were plural points at which the cross-talk value crossed $50 \%$, the farthest one was used. The thick curves in Figs. 4c-f show boundaries of the separation tolerance region when true sources had a y-oriented moment and were 30-mm deep in the medial central area. The cross-talk value was lower than $50 \%$ outside the boundaries. The thin curves in these schematics show the boundaries at which the cross-talk value was 25 and $75 \%$. The separation tolerance region for a point source (Fig. 4C) resembled that for a source distributed on a $40 \times 40-\mathrm{mm}$ square (Fig. $4 \mathrm{~d}$ ), and the boundaries extended most widely when dipole 1 had a location error along the $(\mathrm{t} \perp$ ) direction (Figs. $4 \mathrm{C}$ and $4 \mathrm{~d}$ ) in comparison with the other directions $(t \|)$ in Fig. 4e and $(r)$ in Fig. 4f. Since $(t \perp)$ was the direction along which location error most degraded fittings of dipole 1 as described under Location Error, dipole 2 was given a large moment, resulting in high cross talk. In addition, 


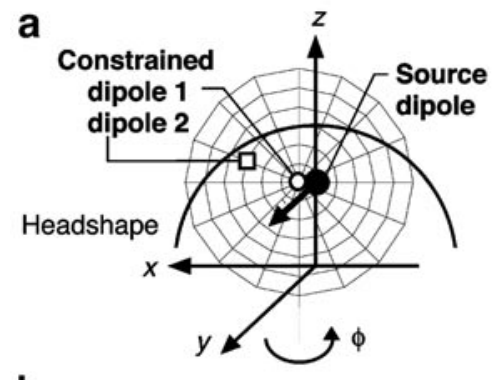

b
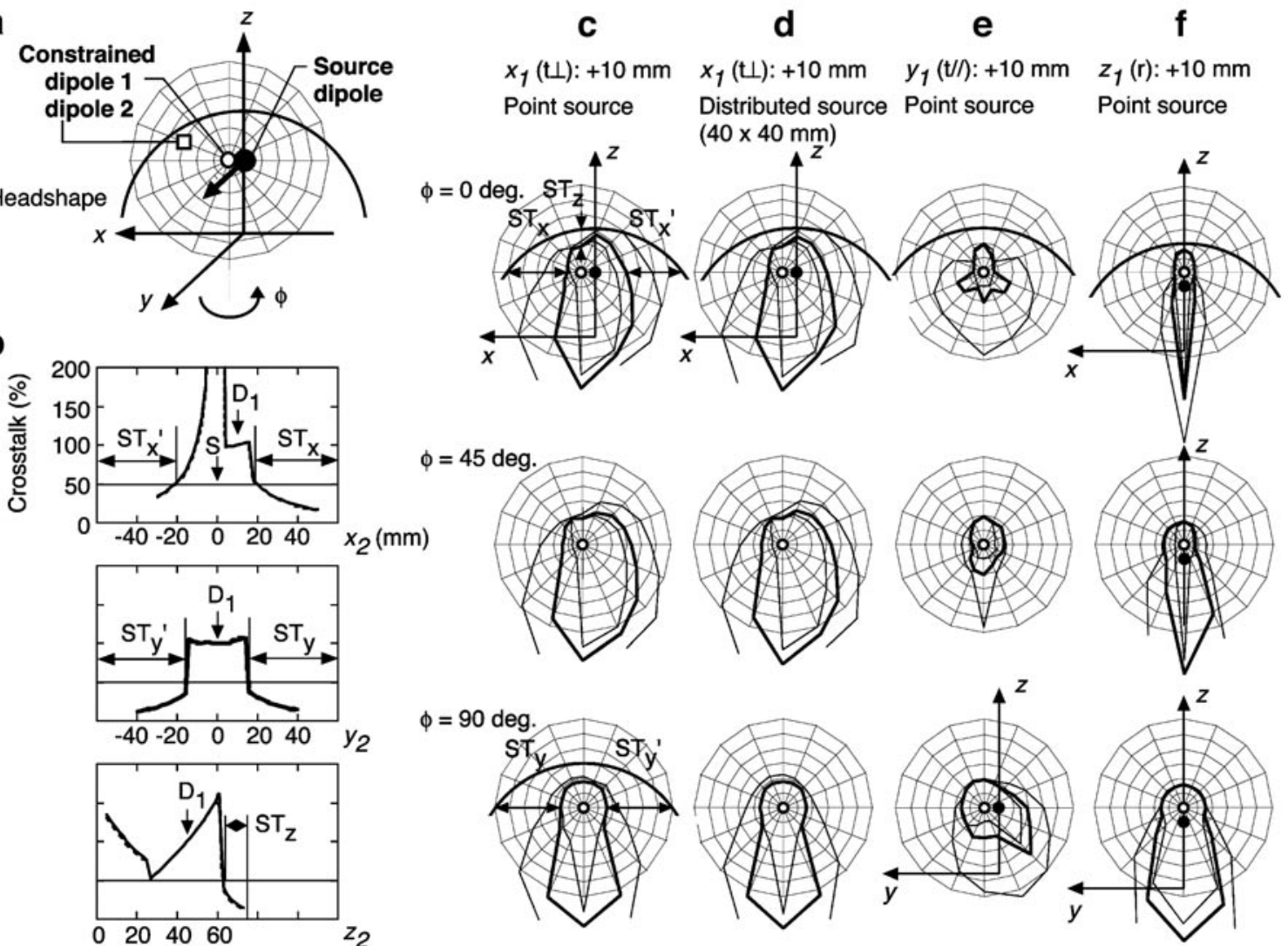

$10 \stackrel{\mapsto}{\mathrm{mm}}$

FIG. 4. (a) Directions in which location of constrained dipole 2 was varied. (b) An example of simulated cross-talk dependence on location for constrained dipole $2,\left(x_{2}, y_{2}, z_{2}\right)$. A true source was $30 \mathrm{~mm}$ deep and had a y-oriented moment, while constrained dipole 1 had a location error of $10 \mathrm{~mm}$ al ong the x axis. The noise was added (solid curve, signal-to-noise ratio of 3) or not (dashed curve) to magnetic fields produced by the source. The symbols S and D1 denote locations of the source and dipole 1, and ST denotes the separation tolerance region in which the cross-talk value was less than 50\%. (c-f) Simulated boundaries of the separation tolerance region in planes with angle $\phi$ of 0,45 , and $90^{\circ}$ (thick curves). Outer and inner thin curves show the boundaries at which the cross-talk threshold was 25 and $75 \%$. Constrained dipole 1 had a location error of $+10 \mathrm{~mm}$ along the $x$ direction for $(\mathrm{c})$ and (d), y direction for (e), and $\mathrm{z}$ direction for (f), and the true source was $30 \mathrm{~mm}$ deep and was a point source for (c), (e), and (f) and a source distributed on a $40 \times 40-\mathrm{mm}$ square for (d).

the boundaries were elongated along the radial direction (Figs. 4c, 4d, and 4f), because it was the direction along which location error least degraded fittings of dipole 2.

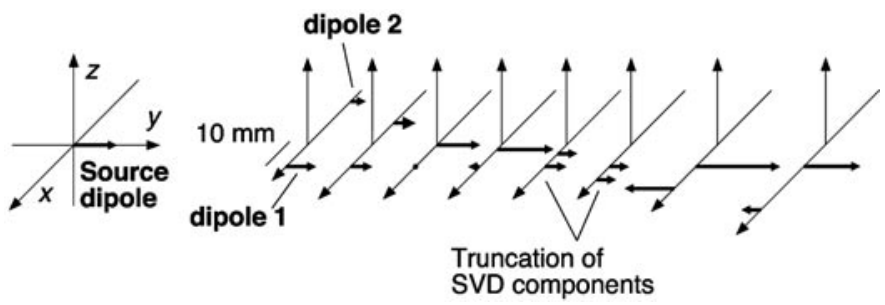

FIG. 5. Moments of two location-constrained dipoles, 1 and 2, fitted to the magnetic fields produced by the source dipole (left). Dipole 1 was shifted by $+10 \mathrm{~mm}$ along the $x$ direction from the source in common, while the location of dipole 2 was varied along the $x$ direction for the eight cases. The origins of the schematics were shifted toward one another (right). The displayed cases were sampled from simulation data shown in the top of Fig. 4(b).

\section{Separation Criteria}

Since we do not know the moment orientations of true sources in actual applications, we cannot evaluate the exact cross-talk value. Instead, we can estimate its probability. Figure 6 shows the probability of an occurrence of high cross talk as a function of the distance between dipole 1 and dipole 2 (solid circles), the distance on the tangential plane (solid triangles), and the distance along the radial direction (solid rectangles). They were calculated using the data simulated for various source areas, orientations, and depths (Table 1) under Effects of Asymmetrical Sensor Layout on Cross Talk. Since the curves were obtained by averaging cases in which dipole 1 had a location error of $10 \mathrm{~mm}$, they show the worst case; if we include dipole 1 nearer to the true sources, the probability curves would be lower. The curve for distance al ong the radial direction was highest among the three curves at distances longer 
- Distance

- Distance in tangential direction

- Distance in radial direction

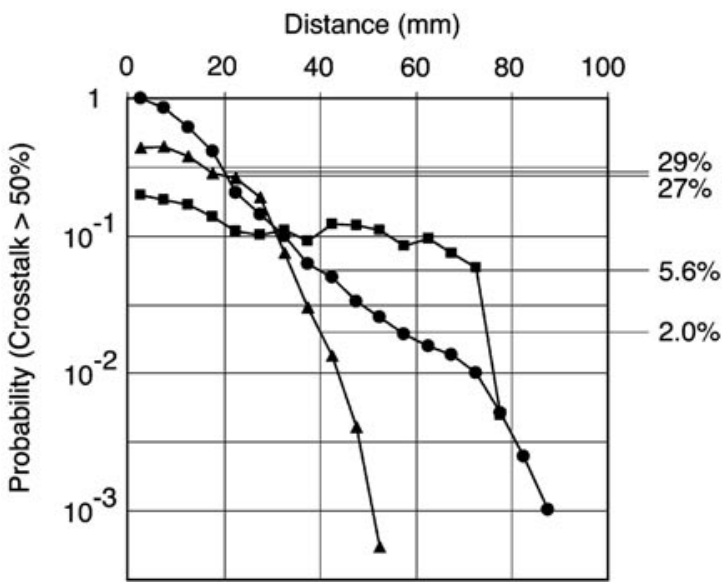

FIG. 6. Simulated probability of an occurrence in which the cross-talk value is higher than $50 \%$ for constrained dipoles 1 and 2 as a function of distance between the dipoles (solid circles), distance in the tangential plane (solid triangles), and distance along the radial direction (solid squares). Dipole 1 has a location error of $10 \mathrm{~mm}$.

than about $30 \mathrm{~mm}$, reflecting the fact that the boundaries of the separation tolerance regions extended largely along the radial direction (Figs. 4c-4f). However, it should be remarked that the order of the three curves was not necessarily the same, because the set of dipoles used in calculating the probability at each distance differed among the three curves. For example, the curve for distance converged to 1 as the distance decreased to 0 , while the curve for distance along the radial direction did not. In the latter case, we calculated the probability using all cases in which dipole 2 was located on a tangential plane, i.e., including dipole 2 that was far from dipole 1 , and showed a small probability. The curve for distance on the tangential plane also included similar cases.

The simplest way to treat high cross talk is to group any pair of dipoles if they are separated by less than a certain criterion. Thus, one may do the following onestep grouping:

(GR1) if any pair of significant dipoles does not satisfy a separation criterion, they are grouped.

Since the probability curve has too low a slope for distance along the radial direction, it is better to use either distance or distance in the tangential plane for this criterion. We consider the following separation criteria:

(SCt40/20) two dipoles are separated by more than $40 / 20 \mathrm{~mm}$ in the tangential plane and

(SCd40/20) two dipoles are separated by more than 40/20 mm.

The separation threshold of $20 \mathrm{~mm}$ is the minimum, because a threshold of less than this value means no grouping for the constrained dipoles, which are sepa- rated by about $20 \mathrm{~mm}$ according to the LC. On the other hand, the threshold of $40 \mathrm{~mm}$ is an example of long-separation thresholds. The probability for high cross talk was 29 and $27 \%$ at the distance of $20 \mathrm{~mm}$ and the distance in a tangential plane of $20 \mathrm{~mm}$ (the thresholds of the shorter criteria (SCd20) and (SCt20)), respectively, while it was as low as 5.6 and $2.0 \%$ at 40 $\mathrm{mm}$ (the thresholds of the longer criteria (SCd40) and (SCt40)) (Fig. 6). In a previous report (Mosher et al., 1993), the authors estimated the errors in localizing a dipole, assuming one or two sources as well as random MEG noise, and found that a second source located as far as $4 \mathrm{~cm}$ from the first source could affect the error. Although the numbers of sources and fitting dipoles differed between the previous and present reports, both studies showed the existence of interference in fittings between dipoles separated by more than $40 \mathrm{~mm}$.

There are two contradictory requirements in our method: grouping the dipoles with high cross talk and avoiding overgrouping (i.e., grouping the dipoles with low cross talk). The longer criteria are better for the former, while the shorter criteria are better for the latter. In this respect, judging similarity between dipole moments in a time course is hel pful to avoid overgrouping, because if two dipoles show different temporal changes, they are independent (low cross talk). Either of the following two conditions can be used for the judgments:

(SL1) two dipoles share significant latencies, i.e., there is at least one latency point at which the moment magnitudes of both dipoles significantly exceed the noise level that is determined in Step 4 under FMRI-Constrained Dipole Procedure; (SL2) the correlation coefficient between moment magnitudes of two dipoles $\left(\mathrm{CC}_{\mathrm{d}}\right)$ is higher than $80 \%$.

The condition (SL 1) is effective when we focus on significant latencies, while the condition (SL2) is more adequate to detect the similarity on average during a time window of interest. We obtained a relationship between the apparent $\mathrm{CC}_{d}$ and the cross talk in Appendix B (Fig. 13) based on an assumption that cross talk is reciprocal and linear for two dipoles. This relation shows that if the cross-talk value is larger than $50 \%$, the apparent $\mathrm{CC}_{\mathrm{d}}$ is expected to be higher than $80 \%$. Although we cannot exclude the case in which two dipoles with low cross talk activate simultaneously $\left(\mathrm{CC}_{\mathrm{d}}>80 \%\right)$, (SL 1/2) conditions can be used to pick up high cross-talk cases.

The one-step grouping has a disadvantage that it may cause a "chaining" phenomenon, which means pairs of neighboring dipoles are grouped in a cascaded way. To suppress the occurrence of the chaining and overgrouping phenomena, we propose the two-step grouping with similarity judgments,

(GR2-1) if any pair of significant dipoles is separated by less than $20 \mathrm{~mm}$ (i.e., they do not satisfy (SCd20)), and show similar temporal changes (SL 1), they are grouped and 
(GR2-2) if any pair of group's centers is separated by less than $40 \mathrm{~mm}$ (i.e., they do not satisfy (SCd40)), and show similar temporal changes (SL2), they are further grouped,

where "significant dipole" means that the magnitude of a dipole moment significantly exceeds a noise level; the judgment procedure is described in Step 4 under FMRIConstrained Dipole Procedure. The criteria (SCd40/20) are used instead of (SCt40/20) in the above grouping because the former are more robust when tangential and radial directions are ambiguous in a real head shape. The first step (GR2-1) is intended to group dipoles that belong to the same cluster of fMRI activation, i.e., a volume consisting of significant voxels connected to each other. The condition (SL1) is chosen to avoid overgrouping in the first step because we especially care for local coincidence of significant latencies for the dipoles in the near neighborhood. A group obtained in (GR2-1) may indude one to a few tens of dipoles depending on activation volumes. If any pairs of groups are judged to suffer high cross talk, they are further grouped in the second step (GR2-2). We restrict the search to groups separated by less than $40 \mathrm{~mm}$, because the probability of finding dipole pairs with high cross talk decreases to as low as 5.6\% at this distance and a wide search may increase possi bilities of occurrence for overgrouping and chaining phenomena. To avoid overgrouping in the second step, we add the condition (SL2), which is adequate to detect global similarity between group moments. Since the probability of high cross talk decreases rapidly as the separation distance exceeds $20 \mathrm{~mm}$, we expect the occurrence of grouping in the second step to be rare.

The probability of high cross talk (Fig. 6) depends on the choice of threshold for cross talk. The present threshold value of $50 \%$ is representative, but one might choose other values. To examine the dependence on threshold, we calculated probability curves for threshold values of 25 and $75 \%$. They also show characteristics similar to those shown in Fig. 6: monotonously decreasing for both distance and distance in the tangential plane, but nearly constant for distance along the radial direction within a range of less than $60 \mathrm{~mm}$. The probabilities for the threshold of $25 \%$ (75\%) were 56 (20) and 53\% (17\%) at a distance of $20 \mathrm{~mm}$ and a distance in the tangential plane of $20 \mathrm{~mm}$, respectively, while they were 21 (1.6) and $18 \%$ $(0.1 \%)$ at $40 \mathrm{~mm}$, and $9.1(0.45)$ and $0.80 \%$ (less than $0.01 \%$ ) at $60 \mathrm{~mm}$. Thus, if we wanted to avoid the low cross talk case (e.g., 25\%) to obtain more independent groups, we would need to increase the separation threshold, e.g., from $20 / 40 \mathrm{~mm}$ in (SCd/t20/40) criteria to about $40 / 60 \mathrm{~mm}$. As a result, the spatial resolution would be come worse because dipoles located in wider areas would be grouped. This indicates a tradeoff between the independence of groups and the spatial resolution in the choice of separation threshold. Since the chaining phenomenon depends on the distribution of $\mathrm{FMRI}$ activation, an empirical finding of the adequate separation threshold would be helpful.

\section{Effects of Asymmetrical Sensor Layout on Cross Talk}

To examine cross talk, the precision of fitted dipole parameters, and their differences due to asymmetry in sensor layout near frontal and lower areas, we carried out simulations using nine combinations of source area/orientation (Table 1) in the same way as those in the medial central area described under Cross Talk. In this section, we restricted the separation tolerance region within a region of interest (ROI) that was the volume within which actual MEG sources could exist. From a structural magnetic resonance image taken from a subject, we determined the ROI as a volume with a radius larger than $22 \mathrm{~mm}$ and a $\mathrm{z}$ coordinate larger than -40 $\mathrm{mm}$ inside a spherical head shape (a radius of $75 \mathrm{~mm}$ ), where the coordinate was as shown in Fig. 1a.

We carried out Kruskal-Wallis tests using one of the four separation criteria (SCt40/20) and (SCd40/20): a test of cross-talk value for constrained dipoles that were located in the ROI and satisfied the separation criterion and tests of normalized moment magnitude and projected angular error of group moment for constrained dipoles that were located in the ROI and did not satisfy the separation criterion, where the group moment was a vector sum of moments for constrained dipoles 1 and 2. The former was intended to test crosstalk values between ungrouped dipoles, while the latter was to test moments of grouped dipoles. The actual groupings by our two-step procedure, (GR2-1) and (GR2-2), would be intermediate between those for (SCd40) and (SCd20). The sum of the number of locations for dipole 2 under test (i.e., the total number of locations in the ROI) was 270,097 .

The test showed that a significant difference occurred for the three quantities among 9 conditions of source area/orientation (Table 1, P $<0.0001$ for each of three quantities) and 10 conditions of error/depth (three or four directions of location errors for dipole 1 (Table 2) and three depths, $P<0.0001$ for each of the three quantities) for each of the four separation criteria.

The differences in the means of the three quantities averaged across the locations of constrained dipoles were larger among error/depth conditions than among area/orientation conditions (middle of Table 4). The former differences showed the following relationships commonly for the four separation criteria and the three source depths (bottom of Table 4),

$$
\begin{aligned}
& \mathrm{av}_{\text {out }}-\text { cross talk } \mathrm{t}_{\perp}>\mathrm{av}_{\text {out }}-\text { cross talk } \mathrm{k}_{\mathrm{t} \|} \\
& \approx \text { av }_{\text {out }} \text {-cross talk } \text {, } \\
& a v_{i n}-m g_{r-}>a v_{i n}-m g_{t \|} \\
& \approx a v_{i n}-m g_{\mathrm{t} \perp}>a v_{i n}-\operatorname{mag}_{\mathrm{r}+}, \\
& a v_{i n} \text {-angle } e_{t \perp}>a v_{i n} \text {-angl } e_{t \|}>a v_{i n} \text {-angle } e_{r} \text {, }
\end{aligned}
$$




\section{TABLE 4}

Grand Average (SD), Maximum Difference in Means of the Three Quantities among the Source Conditions, and Ratio of the Means Averaged across Area/Orientation Conditions to the Grand Average (the Range of the Ratios for the 10 Error/Depth Conditions)

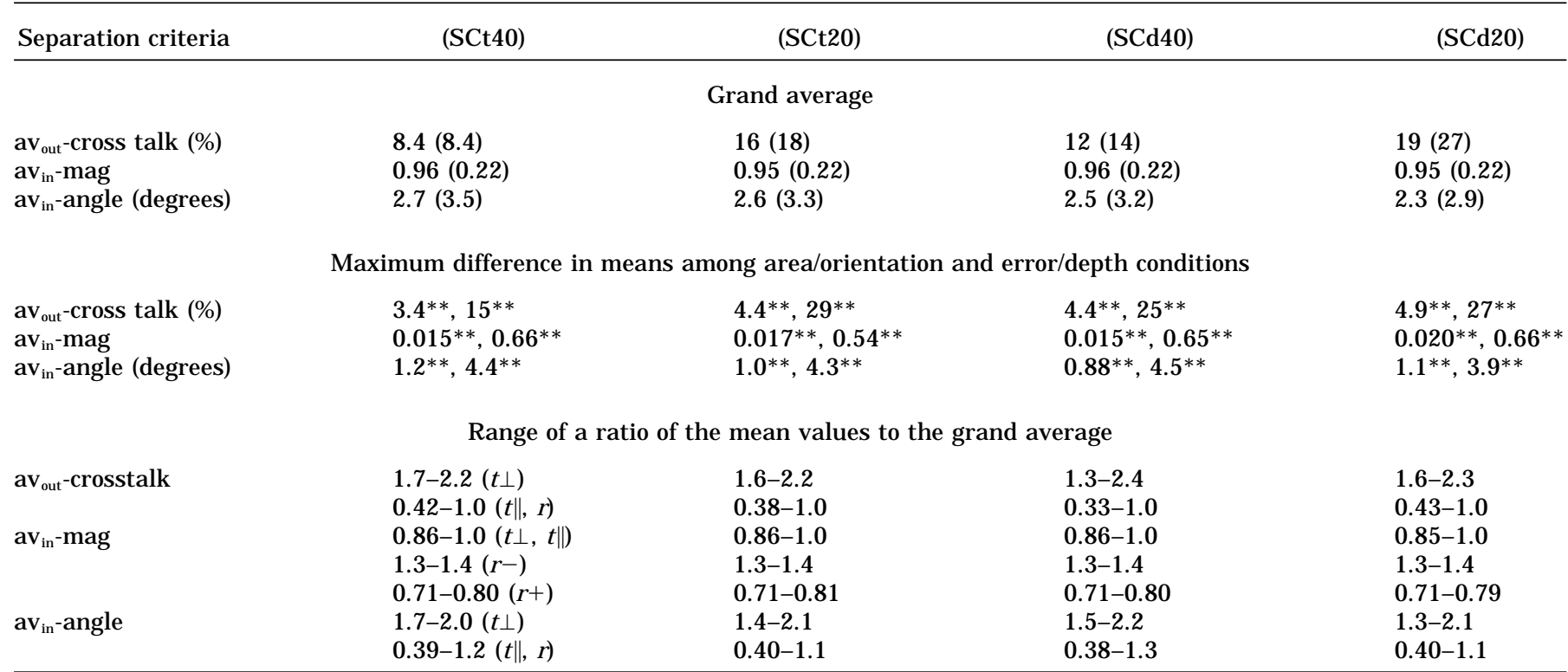

Note Data were evaluated for four separation criteria. A double asterisk denotes that statistical tests showed significant difference among the conditions $(P<0.0001)$. The dependencies of the differences on error conditions are expressed by Eq. (3).

where $\mathrm{av}_{\text {out }}$-cross talk denotes cross-talk value averaged across source area/orientation conditions and constrained dipoles that satisfied a separation criterion (i.e., averaging outside the separation thresholds) in the ROI and $\mathrm{av}_{\mathrm{in}}$-mag and $\mathrm{av}_{\text {in }}$-angle denote normalized moment magnitude and projected angular error of group moment averaged across source area/orientation conditions and constrained dipoles that did not satisfy the criterion (i.e., averaging inside the separation thresholds) in the ROI. The suffixes denote the directions for location error of dipole 1 as summarized in Table 2. For the purpose of obtaining independent dipoles or groups that represent the true sources, it is better that cross talk between ungrouped dipoles, $\mathrm{av}_{\text {out }}{ }^{-}$ cross talk, is smaller while normalized moment magnitude and projected angular error of grouped dipoles, $\mathrm{av}_{\text {in }}$ mag and $\mathrm{av}_{\text {in }}$-angle, are dose to 1 and 0 , respectively.

We carried out the simulations described in this section for only point sources because the three average quantities for point sources were similar to those for uniformly distributed sources. The means (SDs) of $\mathrm{av}_{\text {out }}$-cross talk, $\mathrm{av}_{\text {in }}$-mag, and $\mathrm{av}_{\text {in }}$-angle for the criterion (SCt40) were 8.1(6.8)\%, 1.01(0.24), and $3.1(4.1)^{\circ}$ for a point source, while they were $7.2(6.9) \%$, $1.00(0.24)$, and $2.3(4.2)^{\circ}$ for a source distributed on a $40 \times 40-\mathrm{mm}$ square, when the three quantities were averaged across dipoles for the condition that the sources were $30-\mathrm{mm}$ deep in the medial central area.
The simulation results showed that the $\mathrm{av}_{\text {out }}$-cross talk and $\mathrm{av}_{\text {in }}$-angle had similar dependencies on directions of location error; they were the largest for $(\mathrm{t} \perp$ ) direction and the smallest for the radial direction. This may be related to the fact that location error degraded the fitting of dipole 1 in this same order $(t \perp>t \|>r)$. On the other hand, the dependence of $\mathrm{av}_{\mathrm{in}}$-mag (bottom of Table 4) is considered to reflect a fact that magnetic field strength is inversely proportional to the squared distance between a dipole and a sensor, i.e., moment magnitude is higher as the dipole location is deeper. Although significant differences were shown by the statistical test, the differences in the three quantities among area/orientation conditions were small (middle of Table 4). They may reflect the combined effects of some conditions, such as the direction-dependent characteristics of cross talk, asymmetry in sensor layout, and an exclusion of the inferior and central regions from the ROI. Thus, we need not change the separation criterion nor care for differences in cross talk and fitted dipole parameters among the brain areas.

The grand average for $\mathrm{av}_{\text {out }}$-cross talk, i.e., the average across all area/orientation and error/depth conditions, was larger for (SCt20) than (SCt40) and for (SCd20) than (SCd40) (top of Table 4), because the large cross-talk cases were included in the averaging for the separation threshold of $20 \mathrm{~mm}$ more than for that of $40 \mathrm{~mm}$. 


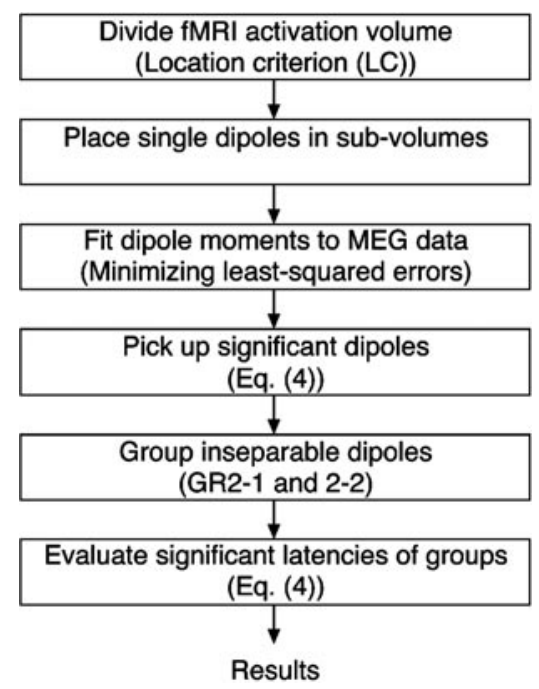

FIG. 7. Procedure for the present analysis method of $\mathrm{fMRI}$-constrained dipoles.

\section{fMRI-C onstrained Dipole Procedure}

Figure 7 shows the present procedure of fMRI-constrained dipole analysis.

\section{Step 1}

We divide an fMRI activation volume into parallelepipeds, instead of the cylinders described under Location Criterion, to avoid overlapping of subvolumes. This procedure can be carried out in the following way: First, we place a rectangular parallelepiped that has a rectangular side parallel to the tangential plane and circumscribes an fMRI activation volume and then, if its diagonal of the rectangular side or the radial side is longer than $20 \mathrm{~mm}$, we divide the parallelepiped by the minimum integer so that the diagonals become shorter than $20 \mathrm{~mm}$. The tangential plane can be determined as a plane perpendicular to a radial line passing through the center of a spherically approximated head shape and the center of mass for the cluster.

\section{Step 2}

Then, we place single dipoles at each center of the divided subvolumes.

\section{Step 3}

We fit moments of the dipoles to MEG data using the ASA software with a real head-shape model. We assume the number of dipoles is less than the number of MEG sensors, so a set of linear equations for the dipole moments is solved using a singular value decomposition so that the solutions satisfy a condition of least squares for errors in magnetic fields.
Step 4

Significant dipoles are judged using the significance level determined in the following way. If we assume moment magnitude $q$ of a constrained dipole fluctuates randomly during a pretrigger period, it is expected to obey the Rayleigh distribution whose cumulative frequency distribution is given by

$$
\begin{aligned}
\Phi(\mathrm{q}) & =1-\mathrm{e}^{-(1 / 2)(\mathrm{q} / \sigma)^{2}}, \\
\sigma & =\sqrt{\frac{2}{\pi}} \overline{\mathrm{q}}
\end{aligned}
$$

where $\bar{q}$ denotes a mean value of $q$ or a noise level. This distribution is the one for a radial component of a moving point in a two-dimensional plane when its components along the two orthogonal axes independently obey a Gaussian distribution with a mean of 0 and a standard deviation of $\sigma$. If we assume that a dipole moment has nearly 2 degrees of freedom on a tangential plane of the real head shape and each component obeys the Gaussian distribution, then its magnitude corresponds to the radial coordinate of the moving point. By using the Bonferroni correction for multiple comparisons (Wiener et al., 1991), we can calculate the uncorrected probability as a corrected probability divided by the independent latency points ( 2 times the frequency bandwidth times the time window of interest) times the number of dipoles. We obtain the significance level $q$ by equating the uncorrected probability and 1 minus $\Phi(q)$ of $E q$. (4) and substituting the noise level $\bar{q}$ during the pretrigger period for each of the fitted constrained dipoles. Then, we judge that a dipole is significant if its moment magnitude exceeds the significance level in latency ranges when $\mathrm{CC}_{m}$ is higher than $94 \%$. We expect that $\mathrm{CC}_{\mathrm{m}}$ satisfies the latter restriction if many constrained dipoles are used in fittings and signal-to-noise ratio is more than 3 , because the mean value of $\mathrm{CC}_{\mathrm{m}}$ averaged across dipole 1 that satisfies the LC is 94\% (Table 3 and Fig. 3). This additional condition is helpful to exclude low-signal cases, i.e., the contributions of a noise or artifact.

\section{Step 5}

We group the significant dipoles with the two-step procedure and obtain a group moment by taking a vector sum of moments for all dipoles included in a group. In the two-step grouping, moment parameters are expected to be between those for the criteria ( $\mathrm{SCd} 20$ ) and (SCd40) summarized in Table 4; the maximum cross-talk value between groups is a value between 12 and 19\%. Although magnitude and orientation of moment for each of dipoles 1 and 2 strongly depend on their locations, moments of their vector sum are similar to those of true sources; the mean value is 
0.95 to 0.96 for normalized moment magnitude and 2.3 to $2.5^{\circ}$ for projected angular error. Thus, grouping helps to make these quantities robust. Nevertheless, when we use these parameters in detailed comparisons, we need to take the ambiguity into account; SD was as large as 0.22 and about $3^{\circ}$ for normalized moment magnitude and projected angular error of group moment, respectively.

\section{Step 6}

We judge significant latencies for groups using Eq. (4) in the same way as described for dipoles in Step 4.

\section{APPLICATION}

To check how effectively the present procedure (Fig. 7) works, we applied it to actual data; we placed constrained dipoles based on fMRI data, fitted their moments to MEG data, detected significant dipoles, and carried out grouping procedures.

\section{Methods}

A set of $\mathrm{AMRI}$ and MEG data was acquired from one subject using a Sternberg paradigm, which was modified to include a cue to prompt phonological rehearsal. The experimental methods were the same as those reported previously (Fujimaki et al., 1999a). In the fMRI experiments, we contrasted neural activation between a test and a control condition. In the test condition, the subject stored six visually presented J apanese katakana (phonogram) characters in memory, executed phonological rehearsals cued by prompts three times, and judged whether a presented character was included with the former ones. The control condition was the same as the test condition except that the stimuli were random dots. The fMRI images were taken at the end of the rehearsal period to contrast the neural activation related to the rehearsal or inner speech in the test condition and that without the rehearsal in the control condition. We took them with a 1.5-T fMRI system (Magnetom Vision, Siemens A.G.) using an echo planar imaging method with parameters TR 12.65 s, TE 66 $\mathrm{ms}$, pixels $2.2 \times 2.2 \mathrm{~mm}$, slice thickness $7 \mathrm{~mm}$, and slice gaps $2.8 \mathrm{~mm}$. In MEG experiments, we recorded neural activation related to inner speech with the same stimuli as the test condition of fMRI. Using the 148channel whole-head MEG system, MEGs were taken from $0.2 \mathrm{~s}$ before to $0.8 \mathrm{~s}$ after the prompt in each epoch. The data were averaged over 144 epochs and were filtered with a bandpass of 0.3 to $40 \mathrm{~Hz}$.

The fMRI data were analyzed using image processing software (MEDx Version 3.20; Sensor Systems, Inc.); they were preprocessed by motion correction and cor egistered to the subject's T1 structural image, which consisted of $4 \times 4 \times 4-\mathrm{mm}$ voxels. After these pro- cesses, the data were smoothed with a spatial filter having full width at half-maximum (FWHM) values of $(8,8,15) \mathrm{mm}$ for the three orthogonal directions, and statistical tests were carried out to judge whether each voxel showed a significant difference in neural activation between the test and the control conditions. In typical analyses of $\mathrm{FMRI}$ data, the FWHM value has usually been chosen to be one to two times the voxel size. For the present data, it was chosen to be larger for the $z$ axis than for the $x$ and $y$ axes because the original slice period was as thick as $9.8 \mathrm{~mm}$ along the $z$ axis (superior-inferior direction). Coordinates of significant resliced voxels were transformed into MEG coordinates. These fMRI and MEG data were processed by the present analysis method described under FMRIConstrained Dipole Procedure.

\section{Results}

In general, fMRI activation areas depend on spatial filtering and a significance level for statistical judgments. We examined their effects on fMRI activation. Figure 8 compares activation maps of the same slice in which colored voxels represent significant activation: the FWHM values of the images in $(x, y, z)$ directions were $(5.1,5.0,8.8) \mathrm{mm}$ for a raw image (Fig. 8a) and $(10.6,10.8,16.8)$ and $(19.9,20.5,28.1) \mathrm{mm}$ for images filtered with FWHMs of $(8,8,15)$ for $(8 b)$ and $(16,16$, 30) $\mathrm{mm}$ for (8c), respectively. The uncorrected probabilities $\left(\mathrm{P}^{\mathrm{u}}\right)$ shown under the graphs were calculated from corrected probabilities ( $\mathrm{P}^{\mathrm{C}}$ ) using a "resel" correction for multiple comparisons (Worsley et al., 1992). Although the same corrected probability threshold $\left(\mathrm{P}^{\mathrm{c}}\right)$ of $1 \%$ was used for all three cases (Figs. 8a- 8c), the activation areas were extended as the FWHM values increased. We know that some suprathreshold voxels are likely to contain neural activity insufficient to exceed the statistical threshold (due to partial voluming or low fMRI-detectable signal), especially those adjacent to superthreshold voxels. To visualize these, we increased the uncorrected probability threshold $\left(P^{\mathrm{u}}\right)$ to as high as $1 \%\left(P^{c} \approx 1\right)$ for nonfiltered data and found that active voxels due to type I error increased (Fig. 8d); however, significant voxels were still gathered in clusters of many adjacent voxels corresponding to the activation clusters obtained with smoothing and stricter thresholds, while intervening areas showed sparser activation. Thus, we decided to use a low-pass filter with FWHM of $(8,8,15) \mathrm{mm}$ to reduce the type I error and adjusted the threshold level to $1 \times 10^{-6}$ for $\mathrm{P}^{\mathrm{u}}$ $\left(P^{c}=5.5 \times 10^{-4}\right)$ so that the active areas (Fig. 8e) resembled the clusters in Fig. 8d.

Figure 9a shows the whole fMRI activation map processed in the same way as in Fig. 8e, and Fig. 9c shows 62 dipoles extracted from the map based on the procedure (Fig. 9b) described in Steps 1 and 2 under FMRIConstrained Dipole Procedure. Six dipoles among the 
a

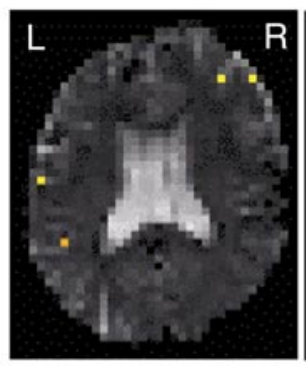

FWHM (mm) No filter
$\mathrm{p}^{\mathrm{u}}$
$\left(p^{c}\right)$

8

a
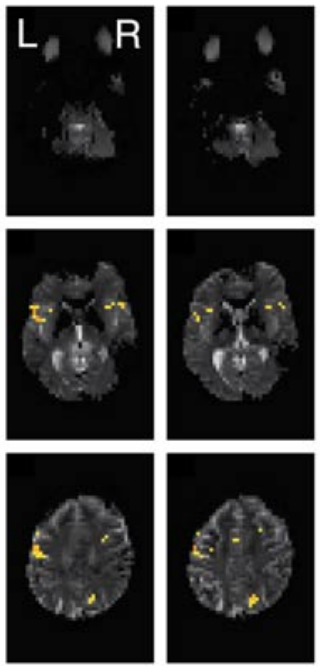

$(8,8,15)$

$1.6 \times 10^{-5}$

$\left(1.0 \times 10^{-2}\right)$
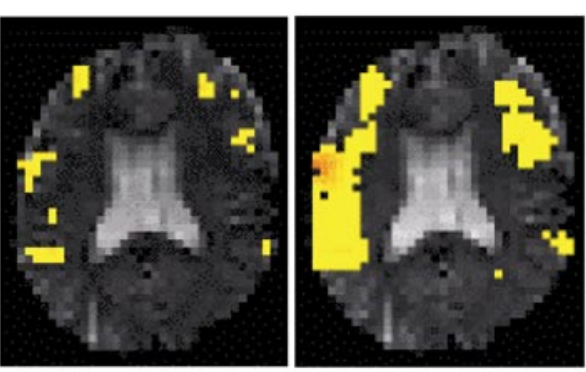

$(16,16,30)$

$9.3 \times 10^{-5}$

$\left(1.0 \times 10^{-2}\right)$
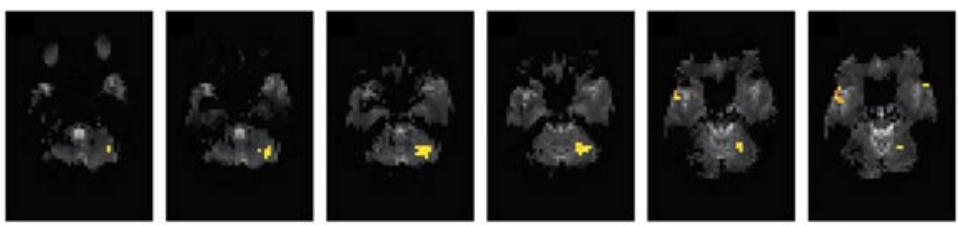

$(8,8,15)$

No filter

$1.0 \times 10^{-2}$

$1.0 \times 10^{-6}$

$(\sim 1.0)$

$\left(5.5 \times 10^{-4}\right)$
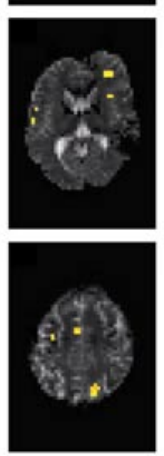
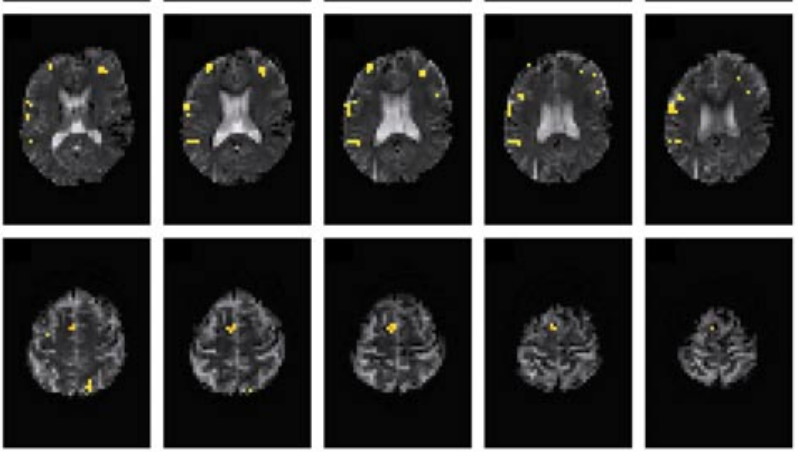

b

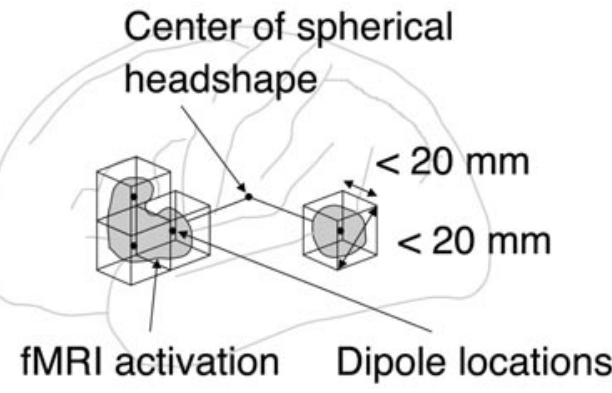

9
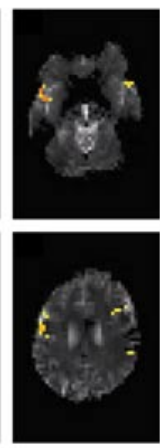

C

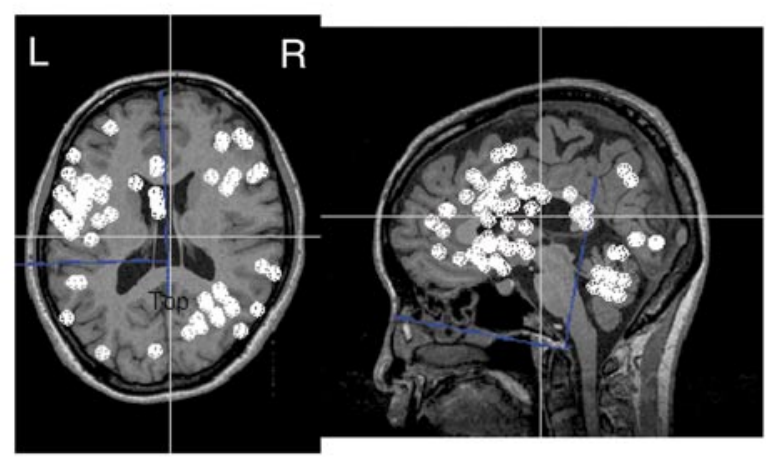

FIG. 8. (a) Transverse slices of $f M R I$ activation measured with a prompted inner speech experiment. They show significant activation in Broca's area, its homologue in the right hemisphere, and the superior temporal areas. The spatial filters were applied with FWHM values shown below the images for (b), (c), and (e), but not for (a) and (d). The significance level for detecting activity was expressed in terms of uncorrected $\left(\mathrm{p}^{\mathrm{u}}\right)$ and corrected $\left(\mathrm{p}^{\mathrm{c}}\right)$ probabilities.

FIG. 9. (a) T1-coregistered fMRI activation map obtained at uncorrected significance level of $1 \times 10^{-6}$. (b) A schematic drawing that shows how to divide fMRI activation volumes in the present method. (c) Locations of 62 dipoles that were located at each center of divided subvolumes to be fitted to MEG data. The locations (white dots) were projected onto the two MRI slices displayed.

62 were added to take into account activation for the control condition that was subtracted from the fMRI activation. In order to find the activation areas for the control condition in which the subject did not perform inner speech when prompted, we conducted another $\mathrm{fMRI}$ experiment contrasting neural activation during 

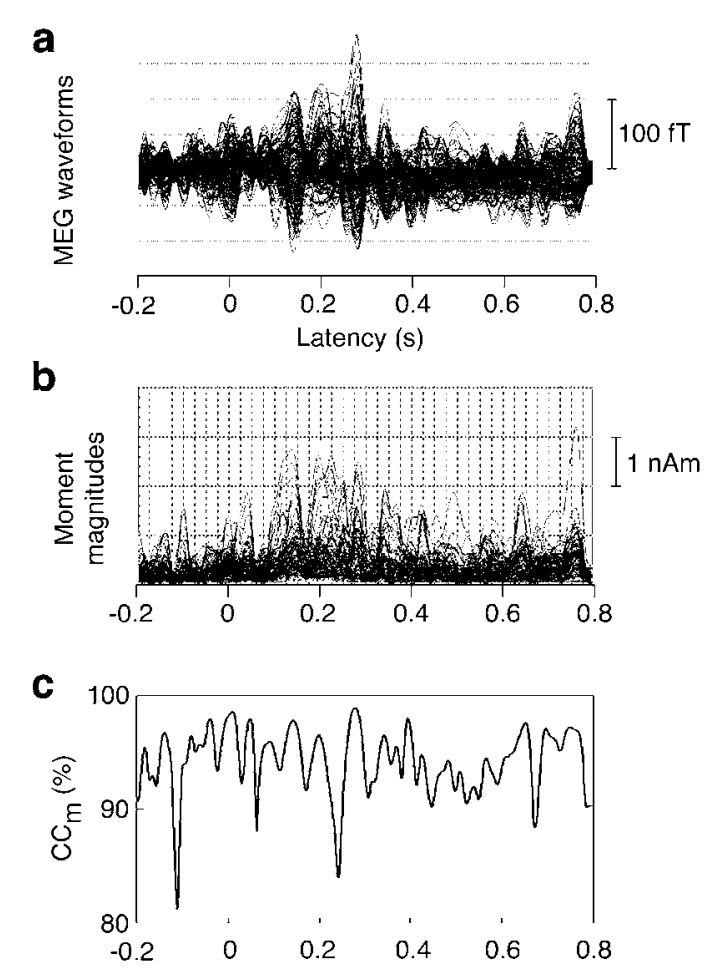

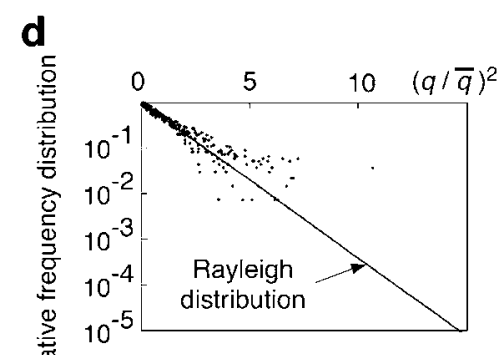

FIG. 10. (a) Average MEG waveforms measured with 148 sensors from one subject using a task of prompted inner speech. (b) Moment magnitudes of the 62 constrained dipoles. (c) $\mathrm{CC}_{\mathrm{m}}$. (d) A cumulative frequency distribution of the normalized moment magnitude during a pretrigger period for the dipoles.

the control condition and that during a rest condition. The six additional dipoles were placed in the occipital extrastriate visual cortices, posterior fusiform gyrus, and posterior inferior temporal areas of both hemispheres. The other activation areas for the control condition were common to those included in the map contrasting the test and control conditions, such as the SMA, cingulate, precentral gyrus, precuneus, parietooccipital sulcus, Broca's area, its right homologue, fusiform gyrus, and cerebellum.

Figure 10(a) shows a superposition of average MEG waveforms for 148 magnetic sensors. Figures 10b and 10c show moment magnitudes of the 62 constrained dipoles and $\mathrm{CC}_{\mathrm{m}}$. Figure $10 \mathrm{~d}$ shows a cumulative frequency distribution for magnitude of dipole moments during the pretrigger period. The data agreed well with the Rayleigh distribution (straight line). The significance level was determined by the distribution and uncorrected probability that were computed in Step 4 under FMRI-Constrained Dipole Procedure using parameters of corrected probability (1\%), frequency bandwidth $(40 \mathrm{~Hz})$, time window of interest $(700 \mathrm{~ms})$, and number of dipoles (62). As a result, 35 dipoles among the 62 were judged to be significant.

Figure 11 shows how the significant dipoles were grouped when we carried out the suboptimal one-step grouping (GR1) by varying the separation threshold for both tangential and radial directions from 20 to $80 \mathrm{~mm}$.
We grouped dipoles if they were separated by less than the threshold value and shared one or more significant latencies when $\mathrm{CC}_{\mathrm{m}}$ exceeded $94 \%$. All dipoles were grouped into one for the threshold value of $80 \mathrm{~mm}$, showing an extreme case of overgrouping in the onestep grouping. F urthermore, when the threshold value was $40 \mathrm{~mm}$, resembling the condition of (SCd40), the "chaining" phenomenon occurred: Broca's area, the insula, the area near the precentral sulcus, and Wernicke's area were grouped into one. In contrast, the two-step grouping showed a clear advantage: The first step resulted in 13 groups. In the second step, no more groupings occurred in this example. Time courses for the magnitudes of group moments are shown in Fig. 12 , in which rectangles indicate significant latencies. To avoid unstable data, we neglected a significant latency range if its duration was shorter than $10 \mathrm{~ms}$.

Although the probability threshold value was chosen by comparing the filtered fMR images with those without filtering (see Methods), there is some arbitrariness in the method. In order to examine how the threshold value and, thus, the spatial extensions covered by dipoles affected the final significant groups, we applied the present method to the same data by varying the threshold value. Table 5 shows the results for the corrected probability threshold values $\left(\mathrm{P}^{\mathrm{c}}\right)$ of $5.5 \times 10^{-4}$ (the same case as shown in Fig. 12), $1.0 \times 10^{-4}$, and $1.0 \times 10^{-2}$. The fMRI activation detected with these 


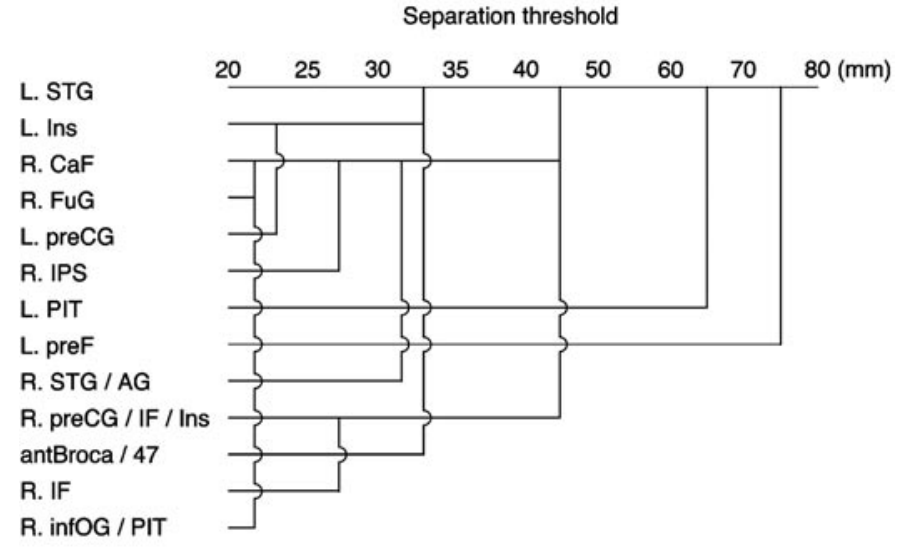

FIG. 11. Diagram showing how dipoles were grouped by the one-step grouping (GR1) while varying a separation threshold common to both tangential and radial directions. The significant dipoles were grouped if they were apart by less than the threshold and shared significant latencies when $\mathrm{CC}_{\mathrm{m}}$ exceeded 94\%. The abbreviations for area names are STG, superior temporal gyrus; Ins, insula; $\mathrm{CaF}$, calcarine fissure; FuG, fusiform gyrus; preCG, precentral gyrus; IPS, intraparietal sulcus; PIT, posterior inferior temporal area; preF, prefrontal area; AG, angular gyrus; IF, inferior frontal area; antBroca, anterior Broca's area; 47, Brodmann's area 47; and infOG, inferior occipital gyrus.

threshold values resulted in 62, 27, and 118 dipoles, respectively. The significant groups and latencies for 27 dipoles were similar to those for 62 dipoles, if we neglect the latencies of groups showing small moment $(<2 \mathrm{nAm})$. Only one major difference in the activation occurred at R. STG-AG, as an fMRI activation was not detected at this location for $\mathrm{P}^{\mathrm{c}}$ of $1.0 \times 10^{-4}$ (27-dipole case).

On the other hand, the 118-dipole case showed more distributed groups with smaller moment magnitudes: two groups were added and two other groups were divided in two. In this case, the number of unknown dipole parameters might exceed the number of measured data (148) as will be discussed later (Discussion). Given this, the dipole fittings were carried out by the minimum norm estimates using ASA software, resulting in broader and smaller solutions. These results showed that too many dipoles (underdetermined cases) are not adequate for the present method.

\section{Discussion}

By applying the present method to a set of actual data, we obtained 13 groups. Among them, the left posterior STG or Wernicke's area, the left insula, and Broca's area were considered specific to the phonological processing and covert articulation (Fujimaki et al., 1999b) that were required in the present experiment. Significant activation was detected at latencies of 122 to $150 \mathrm{~ms}$ and 197 to $209 \mathrm{~ms}$ for the left STG, 122 to 151 ms for the left insula, and 273 to $291 \mathrm{~ms}$ for anterior Broca's area and Brodmann's area 47. Among them, the activation in the left STG and insula was detected at early latencies; however, it is still plausible because the phonological sequences were likely to have been produced before their covert rehearsal was prompted in the present task. Changes in parameters for detecting significant dipoles, such as a corrected probability threshold of $1 \%$ and a $\mathrm{CC}_{\mathrm{m}}$ threshold of $94 \%$, affected the significant latencies but not drastically the groupings, showing the robustness of the results.

Since a dipole with fixed location has 2 degrees of freedom in tangential directions, moments for up to 74 dipoles can be uniquely determined from MEG data of 148 sensors. This does not mean that the 74 dipoles will be independent, because MEG data may have less independent information, and a truncation of singular value decomposition may decrease the degrees of freedom. The number of large eigenvalues for a covariance matrix of magnetic fields has been considered to be the degrees of freedom for signal sources (Mosher et al., 1992). Although we could not find a clear threshold between the large and the small eigenvalues for the present MEG data, there were 26 eigenvalues that exceeded $0.1 \%$ of the maximum value. Roughly speaking, this value was considered to be a ratio of the squared signal for the smallest source component to that for the largest one. Given this, finding 13 groups meant that the present method could detect a source component that was as small as $0.1 \%$ in power (3\% in magnitude) of the maximum one. Furthermore, the ratio was likely to be limited by the inverse of the maximum signal-to-noise ratio of MEG data, which coincided in the present case. These considerations suggest that a small number of final groups, around 10 , seems to be most reliable for reconstructing neural sources from information that MEG data have. In this respect, many dipoles, such as more than a hundred, as other methods have shown (Wagner and Fuchs, 1999; Dale et al., 2000), cannot be fully supported from MEG data.

If $\mathrm{fMRI}$ activation areas extend very widely, the number of dipoles may also become large in the present method, resulting in a grouping of dipoles with large separations due to the chaining effect. Such large fMRI activation areas mean that the information is insufficient to constrain dipole locations. However, the present data showed that typical fMRI activation areas did not show an excessive number of dipoles if we selected an appropriate probability threshold (see Results), suggesting that many actual data sets can be solved by using the present method.

In the present method, each dipole covers neural activity inside a subvolume having a size of $2 \mathrm{~cm}$ at its maximum. This size may be considered large in comparison with the typical confidence length of less than $1 \mathrm{~cm}$ for the traditional MEG analysis method based on a single-dipole approximation. However, this method showed only one dipole at the posterior superior tem- 

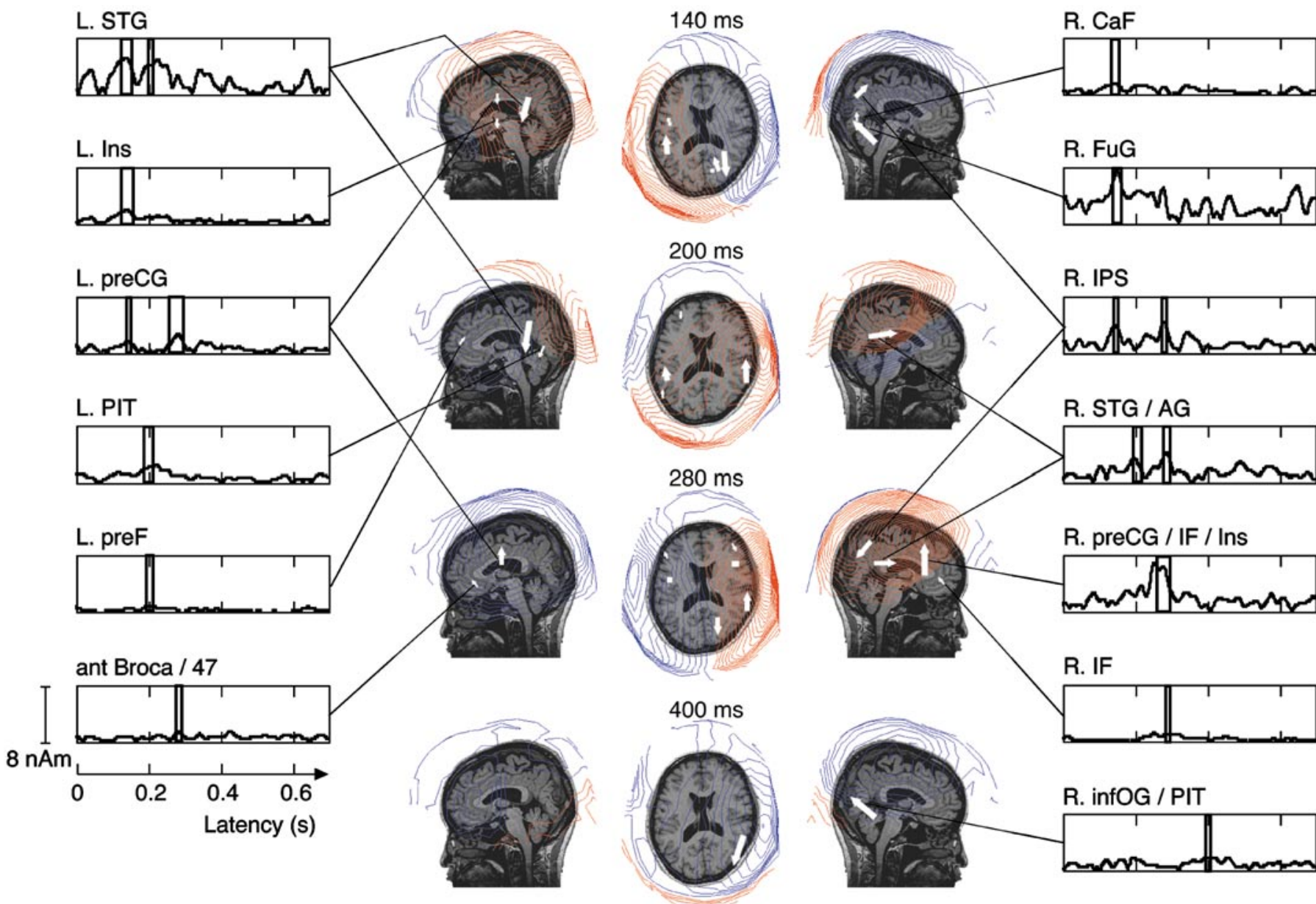

R. IPS

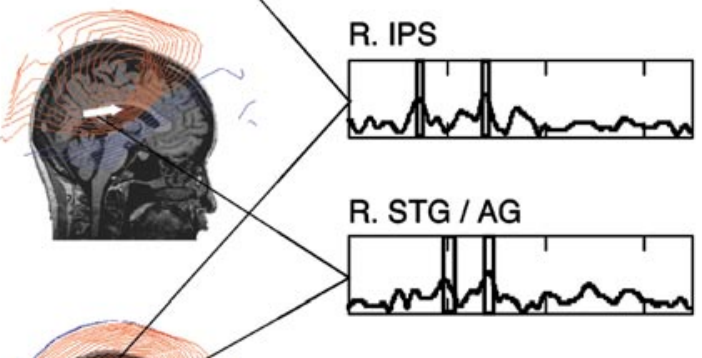

FIG. 12. Locations and time courses of group moments for the 13 groups and magnetic field distributions at the four MEG peaks. The red and blue contour curves drawn by 8-fT steps show positive (i.e., direction of magnetic force lines going out from the brain) and negative (the opposite direction) field distributions, respectively. The white arrows denote group moments projected onto three slices. Their centers show the locations for the group's center-of-gravity, and their lengths are normalized at each latency. Rectangles in the time courses show significant latency ranges. The abbreviations for area names are shown in the legend for Fig. 11.

poral areas in the right hemisphere at a latency of 186 to $194 \mathrm{~ms}$ for the present MEG data, even though we analyzed each of four major peaks using 38 sensor data that covered the major magnetic field distributions (selected from a total of 148). Since the stimulus was the flashing of a small fixation mark, the present data may be especially inadequate for the single-dipole approximation. Our results including 13 significant groups show that the present method is effective for data including many neural sources such as those related to higher cognitive brain functions. The above comparison suggests that the present method sacrifices the accuracy that can be achieved by MEG source localization with less than a few dipoles assumed and, in return, yields approximate but global spatial information on many active sources. In other words, it demonstrates a tradeoff between local and accurate methods on one hand and global and approximate methods on the other hand.
Finally, the present method can be criticized for fixing dipoles only in fMRI activation areas because fMRI-invisible sources cannot be detected. Their detection requires other methods. For example, the fMRIinvisible sources with large contributions may be detected through a reduction in $\mathrm{CC}_{\mathrm{m}}$. Subtraction of magnetic fields produced by constrained dipoles from measured magnetic fields may hel $p$ in detecting them as well. Solutions from multidipole analyses for MEG inverse problems (Ahlfors et al., 1999; Y. Y oshida and $\mathrm{S}$. Ueno, private communication) may help in finding fMRI-invisible dipoles. Furthermore, additional conditions such as cortical constraints (Liu et al., 1998) would also be helpful in refining the present method.

\section{CONCLUSIONS}

We have developed an analysis method using fMRIconstrained dipoles that can reconstruct many dis- 
TABLE 5

Significant Latency Ranges in Units of Milliseconds for the Groups Obtained by the Present FMRI-Constrained MEG Source Analysis Method

Number of dipoles (number of significant fMRI voxels and clusters); $\mathrm{P}^{\mathrm{c}}$

\begin{tabular}{|c|c|c|c|}
\hline \multirow{2}{*}{ Area } & \\
\hline & $62(328,22) ; 5.5 \times 10^{-4}$ & $27(153,17) ; 1.0 \times 10^{-4}$ & $118(814,39) ; 1.0 \times 10^{-2}$ \\
\hline L. Ins & $122-151$ & $104-144$ & $\begin{array}{l}126-145^{*} \\
126-148 *, 181-213^{*}\end{array}$ \\
\hline L. STG & $122-150,197-209$ & $118-148,188-231$ & $(* *), 192-213^{*}$ \\
\hline R. CaF & $131-153^{*}$ & $122-157$ & $143-156 *, 195-212 *$ \\
\hline R. FuG & $135-157$ & $143-157$ & $141-160$ \\
\hline L. preCG & 137-147*, 265-294 & $251-300,370-431$ & $(* * *)$ \\
\hline R. IPS & $138-151,272-287$ & $131-153,272-294$ & (**), 272-287 \\
\hline L. PIT & $187-209$ & $190-231$ & \\
\hline L. preF & 190-209* & $\mathrm{x}$ & $332-351^{*}$ \\
\hline R. STG-AG & $191-203,275-294$ & $x$ & $\begin{array}{l}187-213^{*} \\
191-201,276-290\end{array}$ \\
\hline R. IF-antIns-preCG & $259-294$ & $251-300$ & $253-292$ \\
\hline L. antBroca-47 & 273-291* & $275-287 *, 416-435^{*}$ & $270-291 *, 417-435 *$ \\
\hline R. antIF & $282-294^{*}$ & & \\
\hline R. infOG-PIT & 392-404* & $185-225$ & $397-407 *$ \\
\hline $\begin{array}{l}\text { L. IPS } \\
\text { L. AG }\end{array}$ & $x$ & $x$ & $\begin{array}{l}\text { 140-156*, 197-213*, 279-290* } \\
200-213^{*}\end{array}$ \\
\hline
\end{tabular}

Note Three cases with different probability threshold values $\left(\mathrm{P}^{\mathrm{c}}\right)$ for detecting $\mathrm{fMRI}$ activation, the number of dipoles, and the number of significant fMRI voxels and clusters are shown. The total number of $4 \times 4 \times 4-\mathrm{mm}$ voxels inside the brain was 19,173 . The symbol $X$ denotes nofMRI activation, asterisk denotes small magnitudes ( $<2 \mathrm{nAm})$, and double and triple asterisks denote groups with moment magnitudes slightly lower than the significance threshold at latencies around 140 and 280 ms, respectively. See the legend for Fig. 11 for the area name abbreviations used.

tributed neural sources without using the arbitrary assumption of mathematical criteria such as the minimum norm condition. From simulations of the errors allowed in locating the constrained dipoles and the incorporation of a neighboring dipole in explaining MEG data, we designed the method so as to extract all possible information from the MEG data without creating details in the solution that are not supported by the data. Applying the method to language-experimental data, we demonstrated that it was effective in detecting neural activity with many distributed sources. Additional constraints based on anatomical or physiological grounds could help to refine the present method.

\section{APPENDIX A}

A correlation coefficient, $\mathrm{CC}_{\mathrm{m}}$, is defined as

$$
C_{m}=\frac{\sum_{i}\left(B_{m i}-\overline{B_{m}}\right)\left(B_{c i}-\overline{B_{j}}\right)}{\sqrt{\left[\sum_{i}\left(B_{m i}-\overline{B_{m}}\right)^{2}\right]\left[\sum_{i}\left(B_{c i}-\overline{B_{j}}{ }^{2}\right]\right.}},
$$

where $\mathrm{B}_{\mathrm{mi}}$ and $\mathrm{B}_{\mathrm{c}}$ denote measured and calculated magnetic fields at the ith sensor, $\overline{B_{m}}$ and $\overline{B_{c}}$ denote their means, and summation is carried out for all sensors. We assume that the measured magnetic fields consist of a signal that is produced by true sources and a random noise that has a mean of 0 and is independent between the sensors, and we also assume that the calculated fields are produced by the constrained dipoles, which are fitted to the measured fields. Then, they are expressed as

$$
\begin{aligned}
& B_{m i}=\left(B_{s}+b_{s i}\right)+b_{n i}, \\
& \overline{B_{m}}=B_{s},
\end{aligned}
$$

where $B_{s}$ denotes the mean of the signal, and $b_{s i}$ and $b_{n i}$ denote signal and noise with the mean of 0 . If the constrained dipoles are located near the true sources and are well fitted to their fields, the calculated fields would be approximately equal to the signal,

$$
\begin{aligned}
& \mathrm{B}_{\mathrm{ci}}=\mathrm{B}_{\mathrm{s}}+\mathrm{b}_{\mathrm{si}}, \\
& \overline{\mathrm{B}_{\mathrm{c}}}=\mathrm{B}_{\mathrm{s}} .
\end{aligned}
$$

Then, the following equations can be obtained,

$$
\begin{aligned}
\mathrm{CC}_{\mathrm{m}} & =\frac{\sum_{\mathrm{i}}\left(\mathrm{b}_{\mathrm{si}}+\mathrm{b}_{\mathrm{ni}}\right) \mathrm{b}_{\mathrm{si}}}{\sqrt{\left[\sum_{\mathrm{i}}\left(\mathrm{b}_{\mathrm{si}}+\mathrm{b}_{\mathrm{ni}}\right)^{2}\right]\left[\sum_{\mathrm{i}} \mathrm{b}_{\mathrm{si}}^{2}\right]}}=\frac{1}{\sqrt{1+(\mathrm{S} / \mathrm{N})^{-2}}}, \\
\mathrm{~S} & =\sqrt{\sum_{\mathrm{i}} \mathrm{b}_{\mathrm{si}}^{2}} \\
\mathrm{~N} & =\sqrt{\sum_{\mathrm{i}} \mathrm{b}_{\mathrm{ni}}^{2}}
\end{aligned}
$$




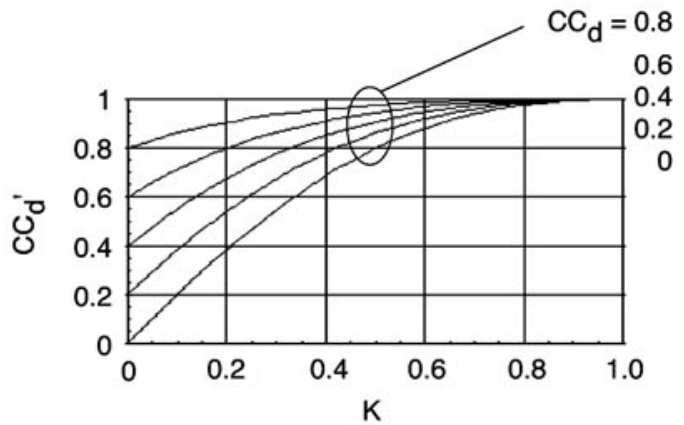

FIG. 13. Relationships (Eq. (B3)) between the apparent and true correlation coefficients of moment magnitude, $\mathrm{CC}_{\mathrm{d}}^{\prime}$ and $\mathrm{CC}_{\mathrm{d}}$, and $\mathrm{a}$ coefficient $\mathrm{K}$ representing an effect of cross talk.

where $\mathrm{S}$ and $\mathrm{N}$ denote the root mean square values for signal and noise.

\section{APPENDIX B}

A correlation coefficient between moment magnitudes of two dipoles, $\mathrm{CC}_{\mathrm{d}}$, is defined as

$$
C C_{d}=\frac{\sum_{j}\left(f_{j}-\bar{f}\right)\left(g_{j}-\bar{g}\right)}{\sqrt{\left[\sum_{j}\left(f_{j}-\bar{f}\right)^{2}\right]\left[\sum_{j}\left(g_{j}-\bar{g}\right)^{2}\right]}},
$$

where $f_{j}$ and $g_{j}$ denote the moment magnitudes of dipoles 1 and 2 at the jth latency point, and summation is carried out for all latency points in the time window of interest. If we assume that $f_{j}$ and $g_{j}$ are independent and cross talk is expressed by a constant coefficient $K$, the moment magnitudes including the effects of cross talk, $f_{j}^{\prime}$ and $g_{j}^{\prime}$, are expressed by

$$
\begin{aligned}
& f_{j}^{\prime}=f_{j}+K g_{j}, \\
& g_{j}^{\prime}=g_{j}+K f_{j} .
\end{aligned}
$$

The apparent correlation coefficient, $\mathrm{CC}_{d}^{\prime}$, is obtained by replacing $f_{j}$ and $g_{j}$ by $f_{j}^{\prime}$ and $g_{j}^{\prime}$ in $E q$. (B1). By substituting Eq. (B2), we obtain the following relationship,

$$
C C_{d}^{\prime}=\frac{2 K+\left(1+K^{2}\right) C C_{d}}{1+K^{2}+2 K C C_{d}}
$$

It is graphically shown in Fig. 13.

\section{ACKNOWLEDGMENTS}

We thank Dr. Frank Zanow and Dr. Michiel van Burik of ANT Software B.V. for their advice regarding the simulations using ASA. We also thank Dr. Keisuke Toyama of Shimadzu Corp., Dr. Mitsuo Kawato of ATR Human Information Processing Research Laboratories, Professor Shinya Kuriki of Hokkaido University, Professor Yoichi Okabe of the University of Tokyo, Dr. Shigeru Hayashi and Dr. Hideo Eda of CRL for their discussions and Dr. Tom Holroyd of
CRL for checking the manuscript. This study was supported by Special Coordination Funds for promoting Science and Technology from the Science and Technology Agency of the J apanese Government.

\section{REFERENCES}

Ahlfors, S. P., Simpson, G. V., Dale, A. M., Belliveau, J. W., Liu, A. K., Korvenoja, A., Virtanen, J., Huotilainen, M., Tootell, R. B. H., Aronen, H. J ., and I Imoniemi, R. J . 1999. Spatiotemporal activity of a cortical network for processing visual motion revealed by MEG and fMRI. J . Neurophysiol. 82: 2545-2555.

Dale, A. M., and Sereno, M. I. 1993. Improved Iocalization of cortical activity by combining EEG and MEG with MRI cortical surface reconstruction: A linear approach. J . Cognit. Neurosci. 5: 162-176.

Dale, A. M., Liu, A. K., Fischl, B. R., Buchner, R. L., Belliveau, J . W., Lewine, J . D., and Halgren, E. 2000. Dynamic statistical parametric mapping: Combining $\mathrm{fMRI}$ and MEG for high-resolution imaging of cortical activity. Neuron 26: 55- 67.

Fujimaki, N., Takahashi, K., and Miyauchi, S. 1998. MEG during judgment of visual characters-A comparison with fMRI. In Proceedings of the 11th International Conference on Biomagnetism (T. Yoshimoto et al., Eds.), pp. 664- 667. Tohoku Univ. Press, Sendai.

Fujimaki, N., Nielsen, M., Hayakawa, T., Kato, M., and Miyauchi, S. 1999a. Neural activity dependent on phonological demands in a verbal working memory task. Neurol mage 9: S919.

Fujimaki, N., Miyauchi, S., Pütz, B., Sasaki, Y., Takino, R., Sakai, K., and Tamada, T. 1999b. Functional magnetic resonance imaging of neural activity related to orthographic, phonological, and lexico-semantic judgments of visually presented characters and words. Hum. Brain Mapp. 8: 44-59.

George, J. S., Anie, C. J ., Mosher, J . C., Schmidt, D. M., Ranken, D. M., Schlitt, H. A., Wood, C. C., Lewine, J . D., Sanders, J . A., and Belliveau, J. W. 1995. Mapping function in the human brain with magnetoencephalography, anatomical magnetic resonance imaging, and functional magnetic resonance imaging. J . Clin. Neurophysiol. 12: 406-431.

Grave de Peralta Menendez, R., Hauk, O., Gonzalez Andino, S., Vogt, H., and Michel, C. 1997. Linear inverse solutions with optimal resolution kernels applied to electromagnetic tomography. Hum. Brain Mapp. 5: 454- 467.

Hämäläinen, M., Hari, R., Ilmoniemi, R. J ., Knuutila, J ., and Lounasmaa, O. V. 1993. Magnetoencephalography-Theory, instrumentation, and applications to noninvasive studies of the working human brain. Rev. Mod. Phys. 65: 413- 497.

Hari, R., J outsiniemi, S.-L., and Sarvas, J . 1988. Spatial resolution of neuromagnetic records: Theoretical calculations in a spherical model. Electroencephalogr. Clin. Neurophysiol. 71: 64-72.

Heinze, H. J ., Mangun, G. R., Burchert, W., Hinrichs, H., Scholz, M., Münte, T. F., Gös, A., Scherg, M., J ohannes, S., Hundeshagen, H., Gazzaniga, M. S., and Hillyard, S. A. 1994. Combined spatial and temporal imaging of brain activity during visual selective attention in humans. Nature 372: 543-546.

I waki, S., and Ueno, S. 1998. Weighted minimum-norm source estimation of magnetoencephalography utilizing the temporal information of the measured data. J . Appl. Phys. 83: 6441- 6443.

Korvenoja, A., Huttunen, J ., Salli, E., Pohjonen, H., Martinkauppi, S., Palva, J . M., Lauronen, L., Virtanen, J ., IImoniemi, R. J ., and Aronen, H. J . 1999. Activation of multiple cortical areas in re sponse to somatosensory stimulation: Combined magnetoencephalographic and functional magnetic resonance imaging. Hum. Brain Mapp. 8: 13-27.

Liu, A. K., Belliveau, J. W., and Dale, A. M. 1998. Spatiotemporal imaging of human brain activity using functional MRI constrained magnetoencephalography data: Monte Carlo simulations. Proc. Natl. Acad. Sci. USA 95: 8945- 8950. 
Matsuura, K., and Okabe, Y. 1995. Selective minimum-norm solution of the biomagnetic inverse problem. IEEE Trans. Biomed. Eng. 42: 608-615.

Mosher, J . C., Lewis, P. S., and Leahy, R. M. 1992. Multiple dipole modeling and localization from spatio-temporal MEG data. IEEE Trans. Biomed. Eng. 39: 541-557.

Mosher, J . C., Spencer, M. E., Leahy, R. M., and Lewis, P. S. 1993. Error bounds for EEG and MEG dipole source localization. Electroencephalogr. Clin. Neurophysiol. 86: 303-321.

Sarvas, J . 1987. Basic mathematical and electromagnetic concepts of the biomagnetic inverse problem. Phys. Med. Biol. 32: 11-22.

Scherg, M., and Berg, P. 1991. Use of prior knowledge in brain electromagnetic source analysis. Brain Topogr. 4: 143-150.

Sekihara, K., and Scholz, B. 1996. Generalized Wiener estimation of three-dimensional current distribution from biomagnetic measurements. IEEE Trans. Biomed. Eng. 43: 281-291.
Uusitalo, M. A., and IImoniemi, R. J . 1997. Signal-space projection method for separating MEG or EEG into components. Med. Biol. Eng. Comput. 35: 135-140.

Wagner, M., and Fuchs, M. 1999. fMRI-constrained current density reconstructions. Neurol mage 9: S202.

Wang, J .-Z., Williamson, S. J ., and Kaufman, L. 1992. Magnetic source images determined by a lead-field analysis: The unique minimum-norm least-squares estimation. IEEE Trans. Biomed. Eng. 39: 665- 675.

Winer, B. J., Brown, D. R., and Michels, K. M. 1991. Statistical Principles in Experimental Design, 3rd ed. McGraw-Hill, New York.

Worsley, K. J., Evans, A. C., Marrett, S., and Neelin, P. 1992. A three-dimensional statistical analysis for rCBF activation studies in the human brain. J. Cereb. Blood Flow Metab. 12: 900-918. 